\title{
Consistent Kaluza-Klein truncations via exceptional field theory
}

\author{
Olaf Hohm ${ }^{a}$ and Henning Samtleben ${ }^{b}$ \\ ${ }^{a}$ Center for Theoretical Physics, Massachusetts Institute of Technology, \\ Cambridge, MA 02139, U.S.A. \\ ${ }^{b}$ Université de Lyon, Laboratoire de Physique, UMR 5672, CNRS, \\ École Normale Supérieure de Lyon, 46, allée d'Italie, F-69364 Lyon cedex 07, France \\ E-mail: ohohm@mit.edu, henning.samtleben@ens-lyon.fr
}

ABSTRACT: We present the generalized Scherk-Schwarz reduction ansatz for the full supersymmetric exceptional field theory in terms of group valued twist matrices subject to consistency equations. With this ansatz the field equations precisely reduce to those of lower-dimensional gauged supergravity parametrized by an embedding tensor. We explicitly construct a family of twist matrices as solutions of the consistency equations. They induce gauged supergravities with gauge groups $\mathrm{SO}(p, q)$ and $\mathrm{CSO}(p, q, r)$. Geometrically, they describe compactifications on internal spaces given by spheres and (warped) hyperboloides $H^{p, q}$, thus extending the applicability of generalized Scherk-Schwarz reductions beyond homogeneous spaces. Together with the dictionary that relates exceptional field theory to $D=11$ and IIB supergravity, respectively, the construction defines an entire new family of consistent truncations of the original theories. These include not only compactifications on spheres of different dimensions (such as $\mathrm{AdS}_{5} \times S^{5}$ ), but also various hyperboloid compactifications giving rise to a higher-dimensional embedding of supergravities with non-compact and non-semisimple gauge groups.

KEYWORds: Flux compactifications, Supergravity Models, Superstring Vacua, M-Theory

ARXIV EPRINT: 1410.8145 


\section{Contents}

1 Introduction 1

$2 \mathrm{E}_{7(7)}$ exceptional field theory $\quad 6$

$\begin{array}{ll}2.1 \text { Bosonic field equations } & 7\end{array}$

$2.2 \mathrm{SU}(8) \times \mathrm{E}_{7(7)}$ geometry and fermions 8

2.3 Solutions of the section condition 11

3 Reduction ansatz $\quad 12$

$\begin{array}{ll}3.1 \text { Generalized Scherk-Schwarz ansatz } & 12\end{array}$

$\begin{array}{lll}3.2 & \text { Consistent truncation, fermion shifts and scalar potential } & 15\end{array}$

4 Sphere and hyperboloid compactifications $\quad 19$

$\begin{array}{lll}4.1 & \mathrm{SL}(n) \text { twist equations } & 19\end{array}$

$\begin{array}{lll}4.2 & \text { Sphere and hyperboloid solutions } & 21\end{array}$

4.2.1 The case $\mathrm{SO}(p, q) \quad 22$

4.2.2 The case $\operatorname{CSO}(p, q, r) \quad 24$

$\begin{array}{lll}4.3 & \mathrm{E}_{6(6)} \text { and } \mathrm{E}_{7(7)} \text { twist equations } & 25\end{array}$

$\begin{array}{lll}4.3 .1 & \mathrm{E}_{6(6)} & 25\end{array}$

$\begin{array}{lll}4.3 .2 & \mathrm{E}_{7(7)} & 27\end{array}$

4.4 The induced space-time metric 28

5 Conclusions $\quad 31$

$\begin{array}{ll}\text { A Projection of the spin connection } & 33\end{array}$

\section{Introduction}

The consistent Kaluza-Klein truncation of higher-dimensional supergravity to lowerdimensional theories is an important and in general surprisingly difficult problem. Here consistent truncation means that any solution of the lower-dimensional theory can be embedded into a solution of the original, higher-dimensional theory. This requires that all coordinate dependence of the internal space is consistently factored out. Due to the non-linearity of the supergravity equations of motion this is a highly non-trivial and often impossible challenge for compactifications on curved backgrounds. Only very few examples are known in which such consistency cannot be attributed to an underlying symmetry argument. The simplest class of consistent truncations are the $T^{n}$ toroidal compactifications in which the internal coordinate dependence is completely dropped, extrapolating the original ideas of Kaluza and Klein [1,2] to higher dimensions. Consistency simply follows 
from the fact that all retained massless fields are singlets under the resulting $\mathrm{U}(1)^{n}$ gauge group. In the context of eleven-dimensional supergravity [3] such reductions give rise to the maximal ungauged (thus abelian) supergravities in lower dimensions [4].

More involved examples are sphere compactifications, the prime example being the compactification of eleven-dimensional supergravity on $\mathrm{AdS}_{4} \times \mathrm{S}^{7}$, leading to maximal $\mathrm{SO}(8)$ gauged supergravity in four dimensions [5]. The required consistency conditions are so non-trivial that in the early days of Kaluza-Klein supergravity this shed serious doubt on the possible consistency of sphere compactifications. For $\mathrm{AdS}_{4} \times \mathrm{S}^{7}$ this consistency was, however, established in [6], with recent improvements in [7-10], employing an $\mathrm{SU}(8)$ invariant reformulation of the original eleven-dimensional theory [11]. Other consistent sphere reductions have been constructed in [12-14], including the compactification on $\operatorname{AdS}_{7} \times \mathrm{S}^{4}$.

An important generalization of the usual compactification scheme was put forward by Scherk and Schwarz [15], relating the internal dimensions to the manifold of a Lie group. More recently, the advances in the understanding of the duality symmetries underlying string and M-theory have nourished the idea to identify generalized geometric (and possibly non-geometric) compactifications as generalized Scherk-Schwarz reductions in some extended geometry [16-21]. In the framework of double field theory [22-27], which makes the $\mathrm{O}(d, d)$ T-duality of string theory manifest, generalized Scherk-Schwarz-type compactifications of an extended spacetime have been discussed in [28-31], see also [32, 33] for reductions to deformations of double field theory. In the M-theory case, analogous ideas have been investigated in [34-39] in the duality covariant formulation of the internal sector of $D=11$ supergravity [40-43].

In this paper, we realize this scenario in full exceptional field theory (EFT) [44-48], which is the manifestly U-duality covariant formulation of the untruncated ten- and elevendimensional supergravities. The theory is formulated on a generalized spacetime coordinatized by $\left(x^{\mu}, Y^{M}\right)$, where we refer to $x^{\mu}$ as 'external' spacetime coordinates, while the $Y^{M}$ describe some generalized 'internal' space with $M, N$ labeling the fundamental representation of the Lie groups in the exceptional series $\mathrm{E}_{d(d)}, 2 \leq d \leq 8$. The fields generically include an external metric $g_{\mu \nu}$, an internal (generalized) metric $\mathcal{M}_{M N}$ and various higher $p$ forms, in particular Kaluza-Klein-like vectors $\mathcal{A}_{\mu}{ }^{M}$ in the fundamental representation and possibly 2-forms and higher forms. With respect to the internal space, all fields are subject to covariant section constraints on the extended derivatives $\partial_{M}$ which imply that fields depend only on a subset of coordinates. There are at least two inequivalent solutions to these constraints: for one the theory is on-shell equivalent to 11-dimensional supergravity, for the other to type IIB, in analogy to type II double field theory [49].

The recent ideas of realizing non-trivial (and possibly non-geometric) compactifications as generalized Scherk-Schwarz compactifications are based on an ansatz for the generalized metric of the form

$$
\mathcal{M}_{M N}(x, Y)=U_{M}^{K}(Y) U_{N}^{L}(Y) M_{K L}(x)
$$

in terms of group-valued twist matrices $U_{M}{ }^{N}$ which capture the $Y$-dependence of the fields. With this ansatz, the $Y$-dependence in the corresponding part of the field equations 
consistently factors out, provided the twist matrices satisfy a particular set of first order differential equations which in full generality take the form

$$
\begin{array}{r}
{\left[\left(U^{-1}\right)_{M}^{P}\left(U^{-1}\right)_{N}{ }^{L} \partial_{P} U_{L}^{K}\right]_{(\mathbb{P})} \stackrel{!}{=} \rho \Theta_{M}{ }^{\boldsymbol{\alpha}}\left(t_{\boldsymbol{\alpha}}\right)_{N}{ }^{K},} \\
\partial_{N}\left(U^{-1}\right)_{M}{ }^{N}-(D-1) \rho^{-1} \partial_{N} \rho\left(U^{-1}\right)_{M} \stackrel{!}{=} \rho(D-2) \vartheta_{M} .
\end{array}
$$

Here, $\rho$ is a $Y$-dependent scale factor, $D$ is the number of external space-time dimensions, $\vartheta_{M}$ and $\Theta_{M}{ }^{\alpha}$ are constant so-called embedding tensors that encode the gauging of supergravity, and $[\cdot]_{(\mathbb{P})}$ denotes projection onto a particular subrepresentation. With this ansatz, the scalar action for $\mathcal{M}_{M N}(x, Y)$ reproduces the scalar potential of gauged supergravity for $M_{K L}(x)$, with the twist matrix $U$ encoding the embedding tensor $\Theta_{M}{ }^{\alpha}$ which parametrizes the lower-dimensional theory [34-36].

In this paper, we extend this scheme to the full exceptional field theory with the following main results

(1) We extend the ansatz (1.1) to the field content of the full exceptional field theory, i.e. to the external metric, vector and $p$-forms. In particular, we find that consistency of the reduction ansatz requires a particular form of the 'covariantly constrained' compensating gauge fields, which are novel fields required in exceptional field theory for a proper description of the degrees of freedom dual to those of the higherdimensional metric. E.g. in $\mathrm{E}_{7(7)}$ exceptional field theory, most of the remaining fields reduce covariantly,

$$
\begin{aligned}
g_{\mu \nu}(x, Y) & =\rho^{-2}(Y) g_{\mu \nu}(x), \\
\mathcal{A}_{\mu}{ }^{M}(x, Y) & =\rho^{-1}(Y) A_{\mu}{ }^{N}(x)\left(U^{-1}\right)_{N}{ }^{M}(Y), \\
\mathcal{B}_{\mu \nu \boldsymbol{\alpha}}(x, Y) & =\rho^{-2}(Y) U_{\boldsymbol{\alpha}}{ }^{\boldsymbol{\beta}}(Y) B_{\mu \nu \boldsymbol{\beta}}(x),
\end{aligned}
$$

with the twist matrix $U$ in the corresponding $\mathrm{E}_{7(7)}$ representation and the scale factor $\rho$ taking care of the weight under generalized diffeomorphisms. In contrast, the constrained compensator field which in the $\mathrm{E}_{7(7)}$ case corresponds to a 2-form $\mathcal{B}_{\mu \nu M}$ in the fundamental representation is subject to a non-standard Scherk-Schwarz ansatz that reads

$$
\mathcal{B}_{\mu \nu M}(x, Y)=-2 \rho^{-2}(Y)\left(U^{-1}\right)_{S}{ }^{P}(Y) \partial_{M} U_{P}^{R}(Y)\left(t^{\boldsymbol{\alpha}}\right)_{R}{ }^{S} B_{\mu \nu \boldsymbol{\alpha}}(x),
$$

relating this field to the 2 -forms $B_{\mu \nu \alpha}$ present in gauged supergravity. The ansatz (1.3) for $A_{\mu}{ }^{M}(x, Y)$ encodes the embedding of all four-dimensional vector fields and their magnetic duals. As such, it includes the recent results of [10] for the $\mathrm{SO}(8)$ sphere compactification, but remains valid for a much larger class of compactifications, in particular, for the hyperboloids which we explicitly construct in this paper. The reduction ansatz for the fermionic fields in the formulation of [48] is remarkably simple, their $Y$-dependence is entirely captured by a suitable power of the scale factor $\rho$. 
We show that with the ansatz (1.1)-(1.4), the field equations of exceptional field theory precisely reduce to the field equations of the lower-dimensional gauged supergravity. Via (1.2), the twist matrix $U$ encodes the embedding tensor $\Theta_{M}{ }^{\alpha}, \vartheta_{M}$ which specifies the field equations of the lower-dimensional gauged supergravity [5052]. In case $\vartheta_{M} \neq 0$, the lower-dimensional field equations include a gauging of the trombone scaling symmetry which in particular acts as a conformal rescaling on the metric [52]. These equations do not admit a lower-dimensional action. Yet, also in this case the generalized Scherk-Schwarz ansatz defines a consistent truncation and we reproduce in particular the exact scalar contributions to the lower-dimensional field equations. For $\vartheta_{M}=0$, the reduction is also consistent on the level of the action and we reproduce the full action of gauged supergravity defined by an embedding tensor $\Theta_{M}^{\alpha}$.

(2) The consistency of the generalized Scherk-Schwarz ansatz being guaranteed by the differential equations (1.2), it remains an equally important task to actually solve these equations. For conventional Scherk-Schwarz compactifications the existence of proper twist matrices is guaranteed by Lie's second theorem, but to our knowledge there is no corresponding theorem in this generalized context. In certain cases, the existence of solutions can be inferred from additional structures on the internal manifold, such as the Killing spinors underlying the original construction of [6] and then [9], or the generalized parallelizability underlying certain coset spaces, such as the round spheres [38]. In this paper, we explicitly construct a family of twist matrices as solutions of (1.2), that via the generalized Scherk-Schwarz ansatz give rise to gauged supergravities with gauge groups $\mathrm{SO}(p, q)$ and $\mathrm{CSO}(p, q, r)$. Geometrically, they describe compactifications on internal spaces given by (warped) hyperboloides $H^{p, q}$ (as first conjectured in [53]), thus extending the applicability of generalized Scherk-Schwarz reductions beyond homogeneous spaces. Our construction is based on the embedding of the linear group $\mathrm{SL}(n)$ into the EFTgroup $\mathrm{E}_{d(d)}$ with the internal coordinates $Y^{M}$ decomposing according to

$$
Y^{M} \longrightarrow\left\{Y^{[A B]}, \ldots\right\}, \quad \text { with } \quad A, B=0, \ldots, n-1
$$

i.e. carrying the antisymmetric representation $Y^{[A B]}$. We then construct a family of $\mathrm{SL}(n)$-valued twist matrices, parametrized by non-negative integers $(p, q, r)$ with $p+q+r=n$, satisfying the $\operatorname{SL}(n)$ version of the consistency conditions (1.2). They depend on a subset of $n-1$ coordinates $y^{i}$, embedded into (1.5) as $y^{i} \equiv Y^{[0 i]}$, such that the section constraint of exceptional field theory is identically satisfied. Upon embedding into $\mathrm{E}_{d(d)}$, these twist matrices turn out to solve the full version of consistency conditions (1.2), provided the number of external dimensions $D$ is related to $n$ as

$$
\frac{1}{2}(D-1)=\frac{n-2}{n-4} .
$$

With the three principal integer solutions $(D, n)=(7,5),(5,6),(4,8)$, we thus obtain solutions of the consistency conditions (1.2) within $\mathrm{SL}(5), \mathrm{E}_{6(6)}$, and $\mathrm{E}_{7(7)}$ EFT. 
Their coordinate dependence is such that the reduction ansatz explicitly satisfies the EFTsection constraints.

Combining these explicit solutions to the consistency equations (1.2) with the generalized Scherk-Schwark ansatz (1.1)-(1.4), we thus define consistent truncations of the full exceptional field theory to lower-dimensional supergravities with gauge groups $\mathrm{SO}(p, q)$ and $\operatorname{CSO}(p, q, r)$. Together with the dictionary that relates exceptional field theory to $D=11$ and IIB supergravity, respectively, (which is independent of the particular choice of the twist matrix $U$ ), the construction thus gives rise to an entire family of consistent truncations in the original theories, including spheres of various dimensions and warped hyperboloids. ${ }^{1}$ Specifically, we compute the internal metric induced by our twist matrices via the Scherk-Schwarz ansatz (1.1), and find

$$
d s^{2}=(1+u-v)^{-2 /(p+q+r-2)}\left(d y^{z} d y^{z}+d y^{a} d y^{b}\left(\delta_{a b}+\frac{\eta_{a c} \eta_{b d} y^{c} y^{d}}{1-v}\right)\right),
$$

with the further split of coordinates $y^{i}=\left\{y^{a}, y^{z}\right\}, a=1, \ldots, p+q-1$, and $z=p+q, \ldots, r$, and the combinations $u \equiv y^{a} y^{a}, v \equiv y^{a} \eta_{a b} y^{b}$. This space is conformally equivalent to the direct product of $r$ flat directions and the hyperboloid $H^{p, q}$. The three integer solutions to (1.6) in particular capture the compactifications around the three maximally supersymmetric solutions $\mathrm{AdS}_{7} \times \mathrm{S}^{4}, \mathrm{AdS}_{5} \times \mathrm{S}^{5}, \mathrm{AdS}_{4} \times \mathrm{S}^{7}$. We stress that in the general case however the metric (1.7) will not be part of a solution of the higher-dimensional field equations. This is equivalent to the fact that the lower-dimensional supergravities in general do not have a critical point at the origin of the scalar potential, as explicitly verified in [53] for the $\mathrm{SO}(p, 8-p)$ supergravities. Nevertheless, in all cases the generalized Scherk-Schwarz ansatz continues to describe the consistent truncation of the higher-dimensional supergravity to the field content and the dynamics of a lower-dimensional maximally supersymmetric supergravity. The construction thus enriches the class of known consistent truncations not only by the long-standing $\mathrm{AdS}_{5} \times \mathrm{S}^{5}$, but also by various hyperboloid compactifications giving rise to non-compact and non-semisimple gauge groups.

Let us stress that throughout this paper we impose the strong version of the section constraint, which implies that locally the fields (i.e. the twist matrix $U$ and scale factor $\rho$ ) depend only on the coordinates of the usual supergravities. ${ }^{1}$ This is indispensable in order to deduce that the consistent truncations from exceptional field theory induce a consistent truncation of the original supergravities. On the other hand, it puts additional constraints on the solutions of (1.2), which makes the actual construction of such solutions a more difficult task. Although naively, one might have thought that for a given embedding tensor $\Theta_{M}{ }^{\alpha}$ a simple exponentiation of $Y^{M} \Theta_{M}{ }^{\alpha} t_{\alpha}$ provides a candidate for a proper twist matrix, the failure of Jacobi identities of the 'structure constants' $\Theta_{M}{ }^{\boldsymbol{\alpha}}\left(t_{\boldsymbol{\alpha}}\right)_{N}{ }^{K}$, and the nontrivial projection in (1.2) put a first obstacle to the naive extrapolation of the Lie algebra

\footnotetext{
${ }^{1}$ It depends on the embedding (1.5) of $\mathrm{SL}(n)$, if the coordinate dependence of the twist matrix falls into the class of eleven-dimensional (IIA) or IIB solutions of the exceptional field theory. This defines in which higher-dimensional theory the construction gives rise to consistent truncations. Unsurprisingly, this is IIA for $D=4,7$, and IIB for $D=5$.
} 
structures underlying the standard Scherk-Schwarz ansatz. On top of this, an object like $Y^{M} \Theta_{M}{ }^{\boldsymbol{\alpha}} t_{\boldsymbol{\alpha}}$ in general violates the section constraint, since $\Theta_{M}{ }^{\boldsymbol{\alpha}}$ in general will have a rank higher than is permitted by the six/seven coordinates among the $Y^{M}$ that are consistent with the section constraint. From this point of view, the standard sphere compactifications take a highly 'non-geometric' form. While we do not expect to encode in this construction genuinely non-geometric compactifications (unless global issues of the type addressed in double field theory in [54] become important), we expect that a proper understanding of highly non-trivial compatifications like for spheres and hyperboloides will be a first step in developing a proper conceptual framework for non-geometric compatifications, which so far are out of reach. It should be evident that the advantage even of the strongly constrained exceptional field theory formulations is a dramatic technical simplification of, for instance, the issues related to consistency proofs, allowing to resolve old and new open questions. In fact, with the full EFTs at hand we can potentially provide a long list of examples of consistent truncations that were previously considered unlikely, such as hyperboloides, warped spheres, compactifications with massive multiplets, etc. Of course, eventually one would like to also include in a consistent framework truly non-geometric compactifications, pointing to a possible relaxation of the strong form of the section constraint.

The rest of this paper is organized as follows. In section 2, we give a brief review of the $\mathrm{E}_{7(7)} \mathrm{EFT}$. Although in this paper most detailed technical discussions will be presented for the $\mathrm{E}_{7(7)}$ EFT the analogous constructions go through for all other EFTs. In section 3 we describe the generalized Scherk-Schwarz ansatz for the full field content of the theory. We show that it defines a consistent truncation of the EFT which reduces to the complete set of field equations of lower-dimensional gauged supergravity with embedding tensor $\Theta_{M}^{\alpha}, \vartheta_{M}$, even in presence of a trombone gauging $\vartheta_{M} \neq 0$. For $\vartheta_{M}=0$, the reduction is also consistent on the level of the action. In section 4 , we construct twist matrices $U$ as explicit solutions of the consistency conditions (1.2). We define a family of $\operatorname{SL}(n)$ twist matrices and show that upon proper embedding into the exceptional groups they solve equations (1.2). The lower-dimensional theories have gauge groups $\mathrm{SO}(p, q)$ and $\mathrm{CSO}(p, q, r)$, respectively. The construction provides the consistent reduction ansaetze for compactifications around spheres $S^{n-1}$ and (warped) hyperboloides $H^{p, q}$. Discussion and outlook are given in section 5 .

\section{$2 \quad E_{7(7)}$ exceptional field theory}

We start by giving a brief review of the $\mathrm{E}_{7(7)}$-covariant exceptional field theory, constructed in refs. $[44,46,48]$ (to which we refer for details). All fields in this theory depend on the four external variables $x^{\mu}, \mu=0,1, \ldots, 3$, and the 56 internal variables $Y^{M}, M=1, \ldots, 56$, transforming in the fundamental representation of $\mathrm{E}_{7(7)}$, however the latter dependence is strongly restricted by the section condition $[40,42,55]$

$$
\left(t_{\boldsymbol{\alpha}}\right)^{M N} \partial_{M} \otimes \partial_{N} \equiv 0, \quad \Omega^{M N} \partial_{M} \otimes \partial_{N} \equiv 0,
$$

where the notation $\otimes$ should indicate that both derivative operators may act on different fields. Here, $\Omega^{M N}$ is the symplectic invariant matrix which we use for lowering and raising 
of fundamental indices according to $X^{M}=\Omega^{M N} X_{N}, X_{N}=X^{M} \Omega_{M N}$. The tensor $\left(t_{\boldsymbol{\alpha}}\right)_{M}{ }^{N}$ is the representation matrix of $\mathrm{E}_{7(7)}$ in the fundamental representation, $\boldsymbol{\alpha}=1, \ldots, 133$. These constraints admit (at least) two inequivalent solutions, in which the fields depend on a subset of seven or six of the internal variables. The resulting theories are the full $D=11$ supergravity and the type IIB theory, respectively.

\subsection{Bosonic field equations}

The bosonic field content of the $\mathrm{E}_{7(7)}$-covariant exceptional field theory is given by

$$
\left\{e_{\mu}{ }^{\alpha}, \mathcal{M}_{M N}, \mathcal{A}_{\mu}{ }^{M}, \mathcal{B}_{\mu \nu \alpha}, \mathcal{B}_{\mu \nu M}\right\}
$$

The field $e_{\mu}{ }^{\alpha}$ is the vierbein, from which the external (four-dimensional) metric is obtained as $g_{\mu \nu}=e_{\mu}{ }^{\alpha} e_{\nu \alpha}$. The scalar fields are described by the symmetric matrix $\mathcal{M}_{M N}$ constructed as $\mathcal{M}_{M N}=\left(\mathcal{V}^{T}\right)_{M N}$ from an $\mathrm{E}_{7(7)}$ valued 56-bein, parametrizing the coset space $\mathrm{E}_{7(7)} / \mathrm{SU}(8)$. Vectors $\mathcal{A}_{\mu}{ }^{M}$ and 2 -forms $\mathcal{B}_{\mu \nu \boldsymbol{\alpha}}$ transform in the fundamental and adjoint representation of $\mathrm{E}_{7(7)}$, respectively. The 2-forms $\mathcal{B}_{\mu \nu N}$ in the fundamental representation describe a covariantly constrained tensor field, i.e. satisfy algebraic equations analogous to $(2.1)$

$$
\begin{aligned}
\left(t_{\boldsymbol{\alpha}}\right)^{M N} \mathcal{B}_{M} \otimes \mathcal{B}_{N}=0, \quad\left(t_{\boldsymbol{\alpha}}\right)^{M N} \mathcal{B}_{M} \otimes \partial_{N}=0 & \\
\Omega^{M N} \mathcal{B}_{M} \otimes \mathcal{B}_{N} & =0, \quad \Omega^{M N} \mathcal{B}_{M} \otimes \partial_{N}=0 .
\end{aligned}
$$

Their presence is necessary for consistency of the hierarchy of non-abelian gauge transformations and can be inferred directly from the properties of the Jacobiator of generalized diffeomorphisms [46]. In turn, after solving the section constraint these fields ensure the correct and duality covariant description of those degrees of freedom that are on-shell dual to the higher-dimensional gravitational degrees of freedom.

The bosonic exceptional field theory is invariant under generalized diffeomorphisms in the internal coordinates, acting via [42]

$$
\mathbb{L}_{\Lambda} \equiv \Lambda^{K} \partial_{K}+12 \partial_{K} \Lambda^{L}\left(t^{\boldsymbol{\alpha}}\right)_{L}^{K} t_{\boldsymbol{\alpha}}+\lambda \partial_{P} \Lambda^{P}
$$

on arbitrary $\mathrm{E}_{7(7)}$ tensors of weight $\lambda$. The weights of the various bosonic fields of the theory are given by

\begin{tabular}{|c|c|c|c|c|c|}
\hline & $e_{\mu}{ }^{\alpha}$ & $\mathcal{M}_{M N}$ & $\mathcal{A}_{\mu}{ }^{M}$ & $\mathcal{B}_{\mu \nu \boldsymbol{\alpha}}$ & $\mathcal{B}_{\mu \nu M}$ \\
\hline$\lambda:$ & $\frac{1}{2}$ & 0 & $\frac{1}{2}$ & 1 & $\frac{1}{2}$ \\
\hline
\end{tabular}

The generalized diffeomorphisms give rise to the definition of covariant derivatives

$$
\mathcal{D}_{\mu}=\partial_{\mu}-\mathbb{L}_{\mathcal{A}_{\mu}},
$$

covariantizing the theory under $x$-dependent transformations (2.4). Their commutator closes into the non-abelian field strengths $\mathcal{F}_{\mu \nu}{ }^{M}$ defined by

$$
\begin{aligned}
\mathcal{F}_{\mu \nu}{ }^{M} \equiv & 2 \partial_{[\mu} \mathcal{A}_{\nu]}{ }^{M}-2 \mathcal{A}_{[\mu}{ }^{N} \partial_{N} \mathcal{A}_{\nu]}{ }^{M}-\frac{1}{2}\left(24\left(t_{\boldsymbol{\alpha}}\right)^{M N}\left(t^{\boldsymbol{\alpha}}\right)_{K L}-\Omega^{M N} \Omega_{K L}\right) \mathcal{A}_{[\mu}{ }^{K} \partial_{N} \mathcal{A}_{\nu]}{ }^{L} \\
& -12\left(t^{\boldsymbol{\alpha}}\right)^{M N} \partial_{N} \mathcal{B}_{\mu \nu \boldsymbol{\alpha}}-\frac{1}{2} \Omega^{M N} \mathcal{B}_{\mu \nu N} .
\end{aligned}
$$


The two-forms $\mathcal{B}_{\mu \nu \boldsymbol{\alpha}}, \mathcal{B}_{\mu \nu N}$ drop out from the commutator $\left[\mathcal{D}_{\mu}, \mathcal{D}_{\nu}\right]$ but are required for $\mathcal{F}_{\mu \nu}{ }^{M}$ to transform covariantly under generalized diffeomorphisms.

The equations of motion of the bosonic theory are most compactly described by a Lagrangian

$$
\begin{aligned}
\mathcal{L}_{\mathrm{EFT}}= & e \widehat{R}+\frac{1}{48} e g^{\mu \nu} \mathcal{D}_{\mu} \mathcal{M}^{M N} \mathcal{D}_{\nu} \mathcal{M}_{M N}-\frac{1}{8} e \mathcal{M}_{M N} \mathcal{F}^{\mu \nu M} \mathcal{F}_{\mu \nu}{ }^{N} \\
& +\mathcal{L}_{\text {top }}-e V\left(\mathcal{M}_{M N}, g_{\mu \nu}\right) .
\end{aligned}
$$

Let us review the different terms. The modified Einstein Hilbert term carries the Ricci scalar $\widehat{R}$ obtained from contracting the modified Riemann tensor

$$
\widehat{R}_{\mu \nu}^{\alpha \beta} \equiv R_{\mu \nu}^{\alpha \beta}[\omega]+\mathcal{F}_{\mu \nu}^{M} e^{\alpha \rho} \partial_{M} e_{\rho}^{\beta}
$$

with the spin connection $\omega_{\mu}^{\alpha \beta}$ obtained from the covariantized vanishing torsion condition $\mathcal{D}_{[\mu} e_{\nu]}^{\alpha} \equiv 0$. Scalar and vector kinetic terms are defined in terms of the covariant derivatives (2.6) and field strengths (2.7). The Lagrangian (2.8) is to be understood as a "pseudo-Lagrangian" in the sense of a democratic action [56], with the vector fields further subject to the first order duality equations

$$
\mathcal{F}_{\mu \nu}^{M}=\frac{1}{2} i e \varepsilon_{\mu \nu \rho \sigma} \Omega^{M N} \mathcal{M}_{N K} \mathcal{F}^{\rho \sigma K},
$$

to be imposed after varying the second-order Lagrangian. The topological term in (2.8) is most compactly given as the boundary contribution of a five-dimensional bulk integral

$$
\int_{\partial \Sigma_{5}} d^{4} x \int d^{56} Y \mathcal{L}_{\text {top }}=\frac{i}{24} \int_{\Sigma_{5}} d^{5} x \int d^{56} Y \varepsilon^{\mu \nu \rho \sigma \tau} \mathcal{F}_{\mu \nu}{ }^{M} \mathcal{D}_{\rho} \mathcal{F}_{\sigma \tau M}
$$

Finally, the last term in (2.8) is given by

$$
\begin{aligned}
V\left(\mathcal{M}_{M N}, g_{\mu \nu}\right)= & -\frac{1}{48} \mathcal{M}^{M N} \partial_{M} \mathcal{M}^{K L} \partial_{N} \mathcal{M}_{K L}+\frac{1}{2} \mathcal{M}^{M N} \partial_{M} \mathcal{M}^{K L} \partial_{L} \mathcal{M}_{N K} \\
& -\frac{1}{2} g^{-1} \partial_{M} g \partial_{N} \mathcal{M}^{M N}-\frac{1}{4} \mathcal{M}^{M N} g^{-1} \partial_{M} g g^{-1} \partial_{N} g \\
& -\frac{1}{4} \mathcal{M}^{M N} \partial_{M} g^{\mu \nu} \partial_{N} g_{\mu \nu}
\end{aligned}
$$

in terms of the internal and external metric.

The full bosonic theory is invariant under vector and tensor gauge symmetries with parameters $\Lambda^{M}, \Xi_{\mu \boldsymbol{\alpha}}, \Xi_{\mu M}$ (the latter constrained according to (2.3)), as well as under generalized diffeomorphisms in the external coordinates. Together, these symmetries uniquely fix all field equations.

\section{$2.2 \mathrm{SU}(8) \times \mathrm{E}_{7(7)}$ geometry and fermions}

In this subsection we review some aspects of the $\mathrm{SU}(8) \times \mathrm{E}_{7(7)}$ covariant geometry, which will be instrumental below, and introduce the fermions of the supersymmetric theory. The geometry, which closely follows that of double field theory $[22,27]$, was developed for the 
fields truncated to the internal sector in $[36,42,43,57]$ and recently completed in [48] for the full $\mathrm{E}_{7(7)}$ exceptional field theory constructed in [46].

We start by introducing a frame formalism, in which the generalized metric is expressed in terms of an $\mathrm{E}_{7(7)}$ valued vielbein $\mathcal{V}$,

$$
\mathcal{M}_{M N}=\mathcal{V}_{M}^{\underline{A}} \mathcal{V}_{N}^{\underline{B}} \delta_{\underline{A B}} \equiv \mathcal{V}_{M i j} \mathcal{V}_{N}^{i j}+\mathcal{V}_{M}^{i j} \mathcal{V}_{N i j}
$$

with flat $\mathrm{SU}(8)$ indices $\underline{A}, \underline{B}, \ldots=1, \ldots, 56$ that split according to the embedding $56=$ $28+28$ as $\underline{A}=\left({ }_{[i j]},{ }^{[i j]}\right), i, j=1, \ldots, 8$. Formulated in terms of $\mathcal{V}$, the theory exhibits a local SU(8) 'tangent space' symmetry. Consequently, one can introduce connections $\mathcal{Q}_{M}$ for this symmetry that can be expressed as

$$
\mathcal{Q}_{M \underline{A}^{\underline{B}}}^{\underline{B}}=\mathcal{Q}_{M i j}{ }^{k l}=\mathcal{Q}_{M[i}{ }^{[k} \delta_{j]}^{l]}
$$

in terms of $\mathrm{SU}(8)$ connections $\mathcal{Q}_{M i}{ }^{j}$ with fundamental indices. We may also introduce a Christoffel-type connection $\Gamma_{M N}{ }^{K}$ and (internal) spin connections $\omega_{M}{ }^{\alpha \beta}$ that render derivatives covariant under generalized diffeomorphisms and local $\mathrm{SO}(1,3)$ transformations, respectively. On a generalized vector $V_{M i}$ transforming in the fundamental of $\mathrm{E}_{7(7)}$ and $\mathrm{SU}(8)$ and as a spinor under $\mathrm{SO}(1,3)$ (whose spinor index we suppress) the covariant derivative is given by

$$
\begin{aligned}
\nabla_{M} V_{N i} \equiv & \partial_{M} V_{N i}+\frac{1}{4} \omega_{M}{ }^{\alpha \beta} \gamma_{\alpha \beta} V_{N i}+\frac{1}{2} \mathcal{Q}_{M i}{ }^{j} V_{N j} \\
& -\Gamma_{M N}{ }^{K} V_{K i}-\frac{2}{3} \lambda(V) \Gamma_{K M}{ }^{K} V_{N i}
\end{aligned}
$$

where $\lambda$ is the density weight of $V$. The internal $\mathrm{SO}(1,3)$ spin connection is given by

$$
\omega_{M}^{\alpha \beta}=e^{\mu[\alpha} \partial_{M} e_{\mu}^{\beta]}
$$

Sometimes it is convenient to work with the combination

$$
\widehat{\omega}_{M}^{\alpha \beta} \equiv \omega_{M}^{\alpha \beta}-\frac{1}{4} \mathcal{M}_{M N} \mathcal{F}_{\mu \nu}{ }^{N} e^{\mu \alpha} e^{\nu \beta},
$$

which naturally enters the supersymmetry variations to be given momentarily.

Next, the remaining connections in (2.15) can be determined (in part) in terms of the physical fields by imposing further constraints. While this does not determine all connections uniquely, the undetermined connection components drop out of all relevant expressions. The first constraint is the generalized torsion constraint, setting to zero a generalized torsion tensor $\mathcal{T}_{M N}{ }^{K}$. In order to state this constraint, note that the Christoffel connection, in its last two indices, takes values in the 133-dimensional Lie algebra of $\mathrm{E}_{7(7)}$, and therefore these connections live in the representation

$$
\Gamma_{M N}{ }^{K}: \quad 56 \otimes 133=\mathbf{5 6} \oplus \mathbf{9 1 2} \oplus \mathbf{6 4 8 0} .
$$

The torsion constraint simply sets the $\mathbf{9 1 2}$ sub-representation to zero,

$$
\mathcal{T}_{M N}{ }^{K}=\left.0 \Longleftrightarrow \Gamma_{M N}{ }^{K}\right|_{\mathbf{9 1 2}}=0
$$


which may be verified to be a gauge covariant condition. Next, demanding that the covariant derivative is compatible with the vierbein density $e=\operatorname{det} e_{\mu}{ }^{\alpha}$ fixes

$$
\nabla_{M} e=0 \quad \Longleftrightarrow \quad \Gamma_{K M}{ }^{K}=\frac{3}{4} e^{-1} \partial_{M} e
$$

allowing for integration by parts with covariant derivatives. This determines the $\mathbf{5 6}$ part of $\Gamma$. It implies, for instance, that the covariant derivative of an $E_{7(7)}$ singlet with density weight $\lambda$ in the fundamental of $\mathrm{SU}(8)$ can be written as

$$
\nabla_{M} V_{i}=e^{\frac{\lambda}{2}} \partial_{M}\left(e^{-\frac{\lambda}{2}} V_{i}\right)+\frac{1}{2} \mathcal{Q}_{M i}{ }^{j} V_{j}
$$

The final constraint is the 'vielbein postulate' stating that the frame field is covariantly constant w.r.t. the combined action of the Christoffel and $\mathrm{SU}(8)$ connection,

$$
\nabla_{M} \mathcal{V}_{N}^{\underline{A}} \equiv \partial_{M} \mathcal{V}_{N}{ }^{\underline{A}}-\mathcal{Q}_{M} \underline{B}^{\underline{A}} \mathcal{V}_{N}^{\underline{B}}-\Gamma_{M N}{ }^{K} \mathcal{V}_{K} \underline{\underline{A}}=0
$$

or, in terms of fundamental $\mathrm{SU}(8)$ indices,

$$
\nabla_{M} \mathcal{V}_{N}^{i j} \equiv \partial_{M} \mathcal{V}_{N}^{i j}+\mathcal{Q}_{M k}{ }^{i i} \mathcal{V}_{N}{ }^{j] k}-\Gamma_{M N}{ }^{K} \mathcal{V}_{K}{ }^{i j}=0
$$

and similarly for its complex conjugate with lower indices.

Let us now give explicit expressions for the determined parts of the spin connections that we will use below. We first note that the vielbein postulate relates the Christoffel connection to the $\mathrm{SU}(8)$ connection as

$$
\Gamma_{\underline{A B}}^{\underline{C}} \equiv\left(\mathcal{V}^{-1}\right)_{\underline{A}}^{M}\left(\mathcal{V}^{-1}\right)_{\underline{B}}^{N} \Gamma_{M N}{ }^{K} \mathcal{V}_{K} \underline{C}=\left(\mathcal{V}^{-1}\right)_{\underline{A}^{M}}\left(\mathcal{V}^{-1}\right)_{\underline{B}}{ }^{N} \partial_{M} \mathcal{V}_{N} \underline{C}-\mathcal{Q}_{A B} \underline{C},
$$

where the indices on $\Gamma$ are 'flattened' by means of the frame field $\mathcal{V}$. Projecting both sides of this equation onto the 912, the generalized torsion constraint (2.19) implies that

$$
\left[\underline{\mathcal{Q}}_{\underline{A B}}^{\underline{C}}\right]_{912}=\left[\left(\mathcal{V}^{-1}\right)_{\underline{A}}^{M}\left(\mathcal{V}^{-1}\right)_{\underline{B}^{N}}^{N} \partial_{M} \mathcal{V}_{N} \underline{C}\right]_{912} .
$$

Thus, while the $\mathbf{9 1 2}$ projection of the Christoffel connection is set to zero by the torsion constraint, the $\mathbf{9 1 2}$ projection of the $\mathrm{SU}(8)$ connection is precisely the part determined by the torsion constraint. Similarly, one obtains an expression for the trace part of $\mathcal{Q}$. Taking the trace of (2.22) yields

$$
\Gamma_{M N}^{M}=\mathcal{V}_{N} \underline{A}^{M}\left(-\partial_{M} \underline{\mathcal{V}}^{M}+\underline{\mathcal{Q}}_{\underline{B}}^{\underline{B}}\right) .
$$

Inserting (2.20) this implies

$$
\underline{\mathcal{Q}}_{\underline{B A}^{\underline{B}}}^{\underline{B}}=\partial_{M} \underline{\mathcal{V}}^{M}+\frac{3}{4} e^{-1} \underline{\mathcal{V}}^{M} \partial_{M} e .
$$

eqs. (2.25) and (2.27) give the full part of the $\mathrm{SU}(8)$ connection that is determined by the above constraints.

We close the discussion of the geometry by giving a definition of the generalized scalar curvature $\mathcal{R}$ that enters the potential. It can be defined through a particular combination of second-order terms in covariant derivatives acting on an $\mathrm{SO}(1,3)$ spinor in 
the fundamental of $\mathrm{SU}(8)$ with density weight $\frac{1}{4}$ (that below will be identified with the supersymmetry parameter),

$$
6 \nabla_{i k} \nabla^{k j} \epsilon_{j}+2 \nabla^{k j} \nabla_{i k} \epsilon_{j}+\nabla^{k l} \nabla_{k l} \epsilon_{i}=\frac{1}{16} \mathcal{R} \epsilon_{i}-\frac{1}{4} \gamma^{\mu \nu} g^{\rho \sigma} \nabla_{i k} g_{\mu \rho} \nabla^{k j} g_{\nu \sigma} \epsilon_{j} .
$$

Here covariant derivative with flattened index are defined as

$$
\nabla^{i j} \equiv\left(\mathcal{V}^{-1}\right)^{i j M} \nabla_{M}
$$

In (2.28) all undetermined connections drop out. Thus, the $\mathcal{R}$ defined by this equation is a gauge scalar, which enters the potential (2.12) as

$$
V=-\mathcal{R}-\frac{1}{4} \mathcal{M}^{M N} \nabla_{M} g^{\mu \nu} \nabla_{N} g_{\mu \nu}
$$

Let us finally turn to the fermions, which are scalar densities under internal generalized diffeomorphisms but transform in non-trivial representations of the tangent space symmetries $\mathrm{SO}(1,3)$ and $\mathrm{SU}(8)$. The gravitino reads $\psi_{\mu}{ }^{i}$, and it has the same weight as the supersymmetry parameter $\epsilon$ :

$$
\lambda\left(\psi_{\mu}{ }^{i}\right)=\lambda(\epsilon)=\frac{1}{4} .
$$

The 56 'spin- $\frac{1}{2}$ ' fermions are given by $\chi^{i j k}$, totally antisymmetric in their SU(8) indices, with density weight

$$
\lambda\left(\chi^{i j k}\right)=-\frac{1}{4}
$$

The supersymmetry variations take the manifestly covariant form written in terms of the above covariant derivatives

$$
\begin{aligned}
\delta_{\epsilon} \psi_{\mu}^{i} & =2 \mathcal{D}_{\mu} \epsilon^{i}-4 \widehat{\nabla}^{i j}\left(\gamma_{\mu} \epsilon_{j}\right), \\
\delta_{\epsilon} \chi^{i j k} & =-2 \sqrt{2} \mathcal{P}_{\mu}{ }^{i j k l} \gamma^{\mu} \epsilon_{l}-12 \sqrt{2} \widehat{\nabla}^{[i j} \epsilon^{k]} .
\end{aligned}
$$

Here $\mathcal{P}_{\mu}$ is the non-compact part of the covariantized Lie algebra valued current $\mathcal{V}^{-1} \mathcal{D}_{\mu} \mathcal{V}$, whose precise form is not important for our purposes in this paper (see [48] for the definition). The projections or contractions of covariant derivatives in these supersymmetry variations are again such that the undetermined connections drop out.

\subsection{Solutions of the section condition}

Even though the fields of (2.8) formally depend on $4+56$ coordinates, their dependence on the internal coordinates is severely restricted by the section constraints (2.1). These constraints admit (at least) two inequivalent solutions, in which the fields depend on a subset of seven or six of the internal variables, respectively, according to the decompositions of the fundamental representation of $\mathrm{E}_{7(7)}$ with respect to the maximal subgroups GL(7) and $\mathrm{GL}(6) \times \mathrm{SL}(2)$, respectively

$$
\begin{aligned}
& \mathbf{5 6} \longrightarrow 7_{+3}+21_{+1}^{\prime}+21_{-1}+7_{-3}^{\prime}, \\
& \mathbf{5 6} \longrightarrow(6,1)_{+2}+\left(6^{\prime}, 2\right)_{+1}+(20,1)_{0}+(6,2)_{-1}+\left(6^{\prime}, 1\right)_{-2} .
\end{aligned}
$$


Upon imposing this restricted coordinate dependence on all fields, the Lagrangian (2.8) (upon proper dualizations and field redefinitions) exactly reproduces the full field equations of $D=11$ supergravity and the type IIB theory, respectively.

The various fields of higher-dimensional supergravity are recovered by splitting the fields (2.2) according to the decompositions (2.34). Their higher-dimensional origin is then most conveniently identified via the GL(1) grading that is captured by the subscripts in (2.34). E.g. the scalar matrix is parametrized as (2.13) in terms of the group-valued vielbein $\mathcal{V}$, parametrized in triangular gauge associated with the GL(1) grading [58] according to

$$
\begin{aligned}
& \mathcal{V}_{\mathrm{IIA}} \equiv \exp \left[\phi t_{(0)}^{\mathrm{IIA}}\right] \mathcal{V}_{7} \exp \left[c_{k m n} t_{(+2)}^{k m n}\right] \exp \left[\epsilon^{k l m n p q r} c_{k l m n p q} t_{(+4) r}\right], \\
& \mathcal{V}_{\mathrm{IIB}} \equiv \exp \left[\phi t_{(0)}^{\mathrm{IIB}}\right] \mathcal{V}_{6} \mathcal{V}_{2} \exp \left[c_{m n a} t_{(+1)}^{m n a}\right] \exp \left[\epsilon^{k l m n p q} c_{k l m n} t_{(+2) p q}\right] \exp \left[c_{a} t_{(+3)}^{a}\right],
\end{aligned}
$$

see [46] for details. The dictionary further requires redefinitions of all the form fields originating from the higher-dimensional $p$-forms in the usual Kaluza-Klein manner, i.e., flattening the world indices with the elfbein and then 'un-flattening' with the vierbein $e_{\mu}{ }^{a}$, as well as subsequent further non-linear field redefinitions and appropriate dualization of some field components, c.f. $[45,46]$.

\section{Reduction ansatz}

In this section we first review the generalized Scherk-Schwarz ansatz of $[16,29,30,32,34$, $36]$. We then extend it to the full field content of the exceptional field theory (2.8) and find in particular, that it requires a non-trivial ansatz for the constrained compensator gauge field $B_{\mu \nu M}$ of (2.3). Together, this defines a consistent truncation of the field equations derived from (2.8).

\subsection{Generalized Scherk-Schwarz ansatz}

The reduction ansatz for the matrix of scalar fields is given by the matrix product

$$
\mathcal{M}_{M N}(x, Y)=U_{M} \underline{\underline{K}}(Y) U_{N} \underline{\underline{L}}(Y) M_{\underline{K L}}(x),
$$

with an $\mathrm{E}_{7(7)}$-valued twist matrix $U_{M^{\underline{N}}}$ satisfying the first order differential equations [36]

$$
\begin{gathered}
{\left[\left(U^{-1}\right)_{\underline{M}}^{P}\left(U^{-1}\right)_{\underline{N}}^{Q} \partial_{P} U_{Q}{ }^{K}\right]_{(912)} \stackrel{!}{=} \frac{1}{7} \rho \Theta_{\underline{M}^{\alpha}}\left(t_{\boldsymbol{\alpha}}\right)_{\underline{N}} \underline{K},} \\
\partial_{N}\left(U^{-1}\right)_{\underline{M}^{N}}-3 \rho^{-1} \partial_{N} \rho\left(U^{-1}\right)_{\underline{M}}^{N} \stackrel{!}{=} 2 \rho \vartheta_{\underline{M}},
\end{gathered}
$$

with a $Y$-dependent factor $\rho(Y)$ and constant tensors $\Theta_{\underline{M}}{ }^{\alpha}$ and $\vartheta_{\underline{M}} \cdot{ }^{2}$ The latter can be identified with the embedding tensor of the gauged supergravity to which the theory reduces

\footnotetext{
${ }^{2}$ With respect to the general form (1.2) of these equations we have introduced a different explicit normalization factor $1 / 7$ (which could be absorbed into $\Theta_{M}{ }^{\alpha}$ ) to achieve later agreement with the $D=4$ formulas. Moreover, for matching the conventions of gauged supergravity [51, 52] we are obliged to perform a rescaling of vector and tensor gauge fields $\sqrt{2} A_{\mu[1103.2785]}^{M}=A_{\mu[\text { here }]}^{M}, B_{\mu \nu \alpha}^{[1103.2785]}=-B_{\mu \nu \alpha}^{[\text {here }]}$, and accordingly of the embedding tensor $\Theta_{M}^{\alpha} \underset{1103.2785]}{\alpha}=\sqrt{2} \Theta_{M}^{\alpha} \underset{\text { [here] }}{\alpha}, \vartheta_{M}^{[1103.2785]}=\sqrt{2} \vartheta_{M}^{[\text {here] }}$. This is most easily seen by comparing the supersymmetry transformation rules from [51, 52] to [48].
} 
after the generalized Scherk-Schwarz ansatz. The notation $[\cdot]_{(912)}$ refers to projection onto the irreducible 912 representation of $\mathrm{E}_{7(7)}$. The density factor $\rho(Y)$ has no analogue in the standard Scherk-Schwarz reduction [15] but ensures that the consistency equations (3.2) transform covariantly under internal generalized diffeomorphisms despite the non-trivial weight carried by the internal derivatives. As can be verified by a direct computation analogous to that proving the covariance of the torsion constraint, the consistency equations are covariant if $U_{M} \underline{\underline{N}}$ is treated as a generalized vector indicated by the index $M$ while the index $\underline{N}$ refers to the global ( $x$ and $Y$ independent) $\mathrm{E}_{7(7)}$ that is preserved by the Scherk-Schwarz reduction ansatz. This is the global $E_{7(7)}$ that is a covariance of the embedding tensor formulation of gauged supergravity (and that, for fixed embedding tensor components $\Theta_{\underline{M}}{ }^{\alpha}$ and $\vartheta_{\underline{M}}$, is broken to the gauge group). In the following we will not indicate the difference between bare and underlined $\mathrm{E}_{7(7)}$ indices since the nature of indices can always be inferred from their position in $U$ and $U^{-1}$.

This Scherk-Schwarz reducution ansatz (3.1) is made such that the action of generalized diffeomorphisms (2.4) with parameter

$$
\Lambda^{M}(x, Y)=\rho^{-1}(Y)\left(U^{-1}\right)_{P}^{M}(Y) \Lambda^{P}(x),
$$

on (3.1) is compatible with the reduction ansatz and induces an action

$$
\delta_{\Lambda} M_{M N}(x)=2 \Lambda^{L}(x)\left(\Theta_{L}^{\boldsymbol{\alpha}}+8 \vartheta_{R}\left(t^{\boldsymbol{\alpha}}\right)_{L}^{R}\right)\left(t_{\boldsymbol{\alpha}}\right)_{\left(M^{P}\right.} M_{N) P}(x),
$$

on the $Y$-independent $M_{M N}$ which is precisely the action of gauge transformations in $D=4$ maximal supergravity with the constant embedding tensor $\Theta_{M}{ }^{\alpha}, \vartheta_{M}$. The second term in (3.4) captures the gauging of the trombone scaling symmetry [59]. The consistency conditions (3.2) together with the section condition (2.1) for the twist matrix imply that $\Theta_{M}{ }^{\alpha}, \vartheta_{M}$ automatically satisfy the quadratic constraints $[51,52]$ that ensure closure of the gauge algebra.

Throughout, we will impose compatibility of the reduction ansatz with the section constraints (2.1). This translates into further conditions on the twist matrix $U_{M}{ }^{N}$. In the conclusions we comment on the possible relaxation of these constraints. In order to describe the consistent truncation of the full exceptional field theory (2.8), the generalized Scherk-Schwarz ansatz (3.1) has to be extended to the remaining fields of the theory which is straightforward for the vierbein, vector and tensor fields as

$$
\begin{aligned}
e_{\mu}{ }^{\alpha}(x, Y) & =\rho^{-1}(Y) e_{\mu}{ }^{\alpha}(x), \\
\mathcal{A}_{\mu}{ }^{M}(x, Y) & =A_{\mu}{ }^{N}(x)\left(U^{-1}\right)_{N}{ }^{M}(Y) \rho^{-1}(Y), \\
\mathcal{B}_{\mu \nu \boldsymbol{\alpha}}(x, Y) & =\rho^{-2}(Y) U_{\boldsymbol{\alpha}}{ }^{\boldsymbol{\beta}}(Y) B_{\mu \nu \boldsymbol{\beta}}(x),
\end{aligned}
$$

with $U_{\boldsymbol{\alpha}} \boldsymbol{\beta}$ denoting the $\mathrm{E}_{7(7)}$ twist matrix evaluated in the adjoint representation. All fields thus transform with the twist matrix $U$ acting on their $\mathrm{E}_{7(7)}$ indices and the power of $\rho^{-2 \lambda}$ corresponding to their weight $\lambda$ under generalized diffeomorphisms (2.4). E.g. the ansatz for the vierbein (which transforms as a scalar of weight $\lambda=\frac{1}{2}$ under (2.4)) ensures that under reduction the action of internal generalized diffeomorphisms consistently reduces to 
the action of the trombone scaling symmetry in $D=4$ supergravity

$$
\begin{aligned}
\delta_{\Lambda} e_{\mu}{ }^{\alpha}(x, Y) & =\Lambda^{M}(x)\left(\partial_{M}\left(\rho^{-1}\right)+\lambda \partial_{M}\left(\rho^{-1}\left(U^{-1}\right)_{N}{ }^{M}\right)\right) e_{\mu}{ }^{\alpha}(x) \\
& =\Lambda^{M}(x) \lambda \rho^{-1}\left(\partial_{M}\left(U^{-1}\right)_{N}{ }^{M}-\left(1+\lambda^{-1}\right) \rho^{-1} \partial_{M} \rho\right) e_{\mu}{ }^{\alpha}(x) \\
& =\Lambda^{M}(x) \vartheta_{M} e_{\mu}{ }^{\alpha}(x) .
\end{aligned}
$$

This confirms that a non-vanishing $\vartheta_{M}$ induces a gauging of the rigid scale invariance (trombone) of the supergravity equations that scales metric and matter fields with proper weights according to (2.5) [59]. The reduction ansatz for the vector field follows (3.3) and ensures that covariant derivatives (2.6) reduce properly:

$$
\begin{aligned}
\mathcal{D}_{\mu} e_{\nu}{ }^{\alpha}(x, Y) & =\rho^{-1}\left(\partial_{\mu}-A_{\mu}{ }^{N} \vartheta_{N}\right) e_{\nu}{ }^{\alpha} \\
\mathcal{D}_{\mu} \mathcal{M}_{M N}(x, Y) & =U_{M}{ }^{P} U_{N}{ }^{Q}\left(\partial_{\mu} M_{P Q}-2 A_{\mu}^{L}\left(\Theta_{L}{ }^{\boldsymbol{\alpha}}+8 \vartheta_{R}\left(t^{\boldsymbol{\alpha}}\right)_{L}{ }^{R}\right)\left(t_{\boldsymbol{\alpha}}\right)_{(M}{ }^{P} M_{N) P}\right) .
\end{aligned}
$$

On the other hand, a consistent reduction of its non-abelian field strength (2.7) requires a non-trivial ansatz for the constrained compensator field $\mathcal{B}_{\mu \nu} M$ according to

$$
\mathcal{B}_{\mu \nu M}=-2 \rho^{-2}\left(U^{-1}\right)_{S}{ }^{P} \partial_{M} U_{P}^{R}\left(t^{\alpha}\right)_{R}^{S} B_{\mu \nu \boldsymbol{\alpha}}
$$

which is manifestly compatible with the constraints (2.3) this field has to satisfy. With this ansatz, it follows that the field strength (2.7) takes the form

$$
\begin{aligned}
& \mathcal{F}_{\mu \nu}{ }^{M}(x, Y)=\rho^{-1}\left(U^{-1}\right)_{N}{ }^{M}\{ 2 \partial_{[\mu} A_{\nu]}{ }^{N}+\Theta_{K}{ }^{\boldsymbol{\alpha}}\left(t_{\boldsymbol{\alpha}}\right)_{L}{ }^{N} A_{[\mu}{ }^{K} A_{\nu]}{ }^{L} \\
&-\frac{1}{3}\left(\Omega^{N P} \Omega_{K L}+4 \delta_{K L}^{N P}\right) \vartheta_{P} A_{[\mu}{ }^{K} A_{\nu]}{ }^{L} \\
&\left.+\left(\Theta^{N \boldsymbol{\alpha}}-16\left(t^{\boldsymbol{\alpha}}\right)^{N K} \vartheta_{K}\right) B_{\mu \nu \boldsymbol{\alpha}}\right\} \\
& \equiv \rho^{-1}\left(U^{-1}\right)_{N}{ }^{M} \mathcal{F}_{\mu \nu}{ }^{N}(x)
\end{aligned}
$$

with the $Y$-independent $\mathcal{F}_{\mu \nu}{ }^{N}(x)$ precisely reproducing the field strength of general gauged supergravity with embedding tensor $\Theta_{M}^{\alpha}$ and $\vartheta_{M}$ [51, 52] (upon the rescaling of footnote 2).

Let us finally note that the consistency equations (3.2) for the twist matrix $U$ are easily generalized to other dimensions as

$$
\begin{array}{r}
{\left[\left(U^{-1}\right)_{M}^{P}\left(U^{-1}\right)_{N}{ }^{L} \partial_{P} U_{L}^{K}\right]_{(\mathbb{P})} \stackrel{!}{=} \rho \Theta_{M}{ }^{\boldsymbol{\alpha}}\left(t_{\boldsymbol{\alpha}}\right)_{N}{ }^{K},} \\
\partial_{N}\left(U^{-1}\right)_{M}{ }^{N}-(D-1) \rho^{-1} \partial_{N} \rho\left(U^{-1}\right)_{M}{ }^{N} \stackrel{!}{=} \rho(D-2) \vartheta_{M},
\end{array}
$$

up to possible normalization factors that can be absorbed into $\Theta_{M}{ }^{\alpha}$ and $\vartheta_{M}$. Here, $D$ denotes the number of external space-time dimensions, and $[\cdot]_{(\mathbb{P})}$ denotes the projection onto the representation of the corresponding embedding tensor in the $D$-dimensional gauged supergravity, c.f. [50]. The coefficients in the second equation can be extracted from (3.6) 
taking into account that the generic weight of the vielbein is $\lambda=\frac{1}{D-2}$. Yet another way of presenting the consistency equations (3.10) is

$$
\begin{aligned}
{\left[\mathcal{X}_{M}^{\boldsymbol{\alpha}}\right]_{(\mathbb{P})} } & \stackrel{!}{=} \rho \Theta_{M}{ }^{\boldsymbol{\alpha}}, \\
\mathcal{X}_{K M}{ }^{K} & \stackrel{!}{=}(1-D) \rho^{-1} \partial_{N} \rho\left(U^{-1}\right)_{M}{ }^{N}+\rho(2-D) \vartheta_{M},
\end{aligned}
$$

with

$$
\mathcal{X}_{M N}{ }^{K} \equiv\left(U^{-1}\right)_{M}{ }^{P}\left(U^{-1}\right)_{N}{ }^{L} \partial_{P} U_{L}{ }^{K} \equiv \mathcal{X}_{M}{ }^{\boldsymbol{\alpha}}\left(t_{\boldsymbol{\alpha}}\right)_{N}{ }^{K} .
$$

The reduction ansatz generalises accordingly: all fields whose gauge parameters transform as tensors under internal generalized diffeomorphisms reduce analogous to (3.5) with the action of the twist matrix $U$ in the corresponding group representation and the factor $\rho^{-(D-2) \lambda}$ taking care of the weight under generalized diffeomorphisms. The additional constrained compensator fields reduce with an ansatz analogous to (3.8). These fields appear among the $(D-2)$-forms of the theory, i.e. among the two-forms in $D=4, \mathrm{E}_{7(7)} \mathrm{EFT}$, c.f. (2.3), and among the vector fields in $D=3, \mathrm{E}_{8(8)}$ EFT. For $D>4$ these fields do not enter the Lagrangian, although they can be defined on-shell through their duality equations.

\subsection{Consistent truncation, fermion shifts and scalar potential}

In the previous subsection we have shown that (external) covariant derivatives and field strengths reduce 'covariantly' under the generalized Scherk-Schwarz reduction, by which we mean that all $\mathrm{E}_{7(7)}$ indices are simply 'rotated' by the twist matrices, up to an overall scaling by $\rho(Y)$ that is determined by the density weight of the object considered. For this to happen it was crucial to include the covariantly constrained compensator 2-form field with its non-standard Scherk-Schwarz ansatz (3.8). From these results it follows that in the action, equations of motion and symmetry variations all $Y$-dependence consistently factors out for all contributions built from external covariant derivatives $\mathcal{D}_{\mu}$. The remaining equations reproduce those of $D$-dimensional gauged supergravity. It remains to establish the same for contributions defined in terms of the internal covariant derivatives $\nabla_{M}$ reviewed in section 2.2. These include the scalar potential (2.12) and the corresponding terms in the supersymmetry variations of the fermions (2.33). In the following we verify that all these terms reduce 'covariantly' as well and show that the reduction precisely reproduces the known scalar potential and fermion shifts in the supersymmetry variations of gauged supergravity.

Before proceeding, let us discuss in a little more detail the consistency of the ScherkSchwarz reduction at the level of the action. In fact, the previous argument only shows that the reduction is consistent at the level of the equations of motion. Consistency at the level of the action requires in addition that the embedding tensor $\vartheta_{M}$ in (3.10) inducing the trombone gauging vanishes. This is in precise agreement with the fact that for lowerdimensional trombone gauged supergravity there is no action principle.

In order to illustrate this point, let us consider the covariant divergence of a generic vector $W^{M}$ of weight $\lambda$ which using (2.20) takes the form

$$
\nabla_{M} W^{M}=\partial_{M} W^{M}+\frac{3}{4}\left(1-\frac{2}{3} \lambda\right) e^{-1} \partial_{M} e W^{M} .
$$


Next we compute the Scherk-Schwarz reduction of this divergence. Here and in the following it will be convenient to use the bracket notation \langle\rangle to indicate that an object is subjected to the Scherk-Schwarz reduction. Recalling $\langle e\rangle=\rho^{-4} e$, we compute

$$
\begin{aligned}
\left\langle\nabla_{M} W^{M}\right\rangle & =\partial_{M}\left(\rho^{-2 \lambda}\left(U^{-1}\right)_{N}{ }^{M}\right) W^{N}+\frac{3}{4}\left(1-\frac{2}{3} \lambda\right) \rho^{4} \partial_{M} \rho^{-4} \rho^{-2 \lambda}\left(U^{-1}\right)_{N}{ }^{M} W^{N} \\
& =\rho^{-2 \lambda}\left[\partial_{M}\left(U^{-1}\right)_{N}{ }^{M}-3 \rho^{-1} \partial_{M} \rho\left(U^{-1}\right)_{N}{ }^{M}\right] W^{N} \\
& =2 \rho^{1-2 \lambda} \vartheta_{M} W^{M},
\end{aligned}
$$

using (3.2) to identify the trombone embedding tensor $\vartheta_{M}$. On the other hand, calculating the equations of motion from the Lagrangian requires partial integration with general currents $J^{M}$ of weight $-1 / 2$

$$
\int \partial_{M}\left(e J^{M}\right)=\int e \nabla_{M} J^{M}
$$

whose boundary contribution is neglected, in obvious contradiction with (3.14) unless $\vartheta_{M}=$ 0 . For non-vanishing trombone parameter $\vartheta_{M}$, the Scherk-Schwarz ansatz thus continues to define a consistent truncation on the level of the equations of motion, however the lowerdimensional equations of motion do not allow for an action principle due to the ambiguities arising from (3.15). The resulting structure corresponds to the trombone gaugings of [52, 59]. This is the analogue of the unimodularity condition $f_{M N}{ }^{M}=0$ to be imposed in standard Scherk-Schwarz reductions [15] for invariance of the measure, c.f. (3.11). Also in that case a non-vanishing $f_{M N}{ }^{M} \equiv \vartheta_{N}$ does in fact not spoil the consistency of the reduction ansatz but just the existence of a lower-dimensional action.

Let us now return to the more general discussion of the Scherk-Schwarz reduction of the internal covariant derivatives $\nabla_{M}$. We begin by applying the generalized Scherk-Schwarz compactification to the $\mathrm{SU}(8)$ connections. Applied to the projection (2.25) of the $\mathrm{SU}(8)$ connection we obtain

$$
\begin{aligned}
\left\langle\left[\underline{\mathcal{Q}}_{\underline{A B}}{ }^{\underline{C}}\right]_{912}\right\rangle & =\left(\mathcal{V}^{-1}\right)_{\underline{A}^{M}}\left(\mathcal{V}^{-1}\right)_{\underline{B}^{N}} \mathcal{V}_{K} \underline{C}\left[\left(U^{-1}\right)_{M}{ }^{M}\left(U^{-1}\right)_{N}{ }^{N} \partial_{M} U_{N}{ }^{K}\right]_{912} \\
& =\frac{1}{7} \rho\left(\mathcal{V}^{-1}\right)_{\underline{A}}^{M}\left(\mathcal{V}^{-1}\right)_{\underline{B}^{N}} \mathcal{V}_{K}{ }^{\underline{C}} \Theta_{M}{ }^{\boldsymbol{\alpha}} t_{\boldsymbol{\alpha} N}{ }^{K}
\end{aligned}
$$

upon using (3.2). This expression features the flattened version of the embedding tensor, also known as the 'T-tensor' [5] in gauged supergravity. Similarly, from (2.27) one finds that the determined (trace) part in the $\mathbf{5 6}$ reduces as,

$$
\left\langle\mathcal{Q}_{\underline{B A}} \underline{B}\right\rangle=\left(\mathcal{V}^{-1}\right)_{\underline{A}}^{N}\left(\partial_{M}\left(U^{-1}\right)_{N}{ }^{M}-3 \rho^{-1}\left(U^{-1}\right)_{N}{ }^{M} \partial_{M} \rho\right)=2 \rho\left(\mathcal{V}^{-1}\right)_{\underline{A}}^{N} \vartheta_{N},
$$

identifying it with the T-tensor corresponding to the trombone embedding tensor. Thus, the parts of the $\mathrm{SU}(8)$ connection that are determined geometrically by generalized torsion and metricity constraints, upon Scherk-Schwarz reduction naturally identify with the Ttensor. Comparing with the definitions of $[51,52]$, the relation is explicitly given by

$$
\left\langle\left[\underline{\mathcal{Q}}_{\underline{A B}} \underline{\underline{C}}\right]_{912}\right\rangle=\frac{2}{7} \rho T_{\underline{A B}}^{\underline{C}}, \quad\left\langle\underline{\mathcal{Q}}_{\underline{B A}}^{\underline{B}}\right\rangle=-2 \rho T_{\underline{A}} .
$$


These relations makes the following comparison with gauged supergravity straightforward.

In order to perform that comparison in detail we note that the $\mathbf{9 1 2}$ representation of the T-tensor decomposes under $\mathrm{SU}(8)$ as

$$
T_{\underline{A B}}^{\underline{C}}: \quad 912 \rightarrow 420+36+\text { c.c. }
$$

which implies, for instance, that the component $T^{i j}{ }_{k l}{ }^{p q}$ of $T_{\underline{A B}} \underline{C}$ can be parametrized as

$$
T_{k l}^{i j}{ }^{p q}=\frac{2}{3} \delta_{\left[k^{[p}\right.} T_{l]}^{q] i j},
$$

in terms of a tensor $T_{i}{ }^{j k l}$, which in turn can be decomposed as

$$
T_{i}^{j k l}=-\frac{3}{4} A_{2 i}{ }^{j k l}-\frac{3}{2} A_{1}{ }^{j[k} \delta^{l]}{ }_{i} .
$$

Here $A_{1}{ }^{i j}$ is symmetric and hence lives in the $\mathbf{3 6}$, and $A_{2}$ satisfies $A_{2 i}{ }^{j k l}=A_{2 i}{ }^{[j k l]}, A_{2 i}{ }^{i k l}=$ 0 , and hence lives in the $\mathbf{4 2 0}$. The tensors $A_{1}$ and $A_{2}$ thus defined contribute to the fermion shifts in the supersymmetry variations and the scalar potential of gauged supergravity. Similarly, the trombone T-tensor, i.e., the flattening of $\vartheta_{M}$, decomposes as

$$
T_{\underline{A}} \equiv\left(\mathcal{V}^{-1}\right) \underline{A}^{M} \vartheta_{M}=\left(T_{i j}, T^{i j}\right) \equiv\left(B_{i j}, B^{i j}\right),
$$

in terms of an antisymmetric tensor $B_{i j}$ (and its complex conjugate). Via eqs. (3.21) and (3.22) and the relations (3.18) for the Scherk-Schwarz reduction of the connections we can express the latter in terms of the gauged supergravity tensors. There is the following subtlety, however: The components of the SU(8) connection entering, say, the fermionic supersymmetry variations are the $\mathcal{Q}_{M i}{ }^{j}$ from (2.14), while the $\mathbf{9 1 2}$ projection in (3.18) shuffles its components around. A slightly technical group-theoretical analysis, whose details we defer to the appendix, shows that the net effect is a rescaling of the $A_{2}$ contribution in the T-tensor by $\frac{7}{3}$ (see (A.10) in the appendix), while $A_{1}$ is untouched. We then find

$$
\begin{aligned}
\left\langle\left[\mathcal{Q}^{i j}{ }_{k m}^{l n}\right]\right\rangle= & \frac{2}{7} \rho\left(-\frac{1}{2} \cdot \frac{7}{3} \delta_{\left[k^{[l} A_{2 m]}{ }^{n] i j}-\delta_{k m}{ }^{[i[l} A_{1}{ }^{n] j]}\right)}\right. \\
& -\frac{16}{27} \rho \delta_{k m}{ }^{[i[l} B^{n] j]}-\frac{2}{27} \rho \delta_{k m}{ }^{l n} B^{i j}
\end{aligned}
$$

where the square bracket indicates projection onto the determined part of the connections. In particular, we obtain

$$
\begin{aligned}
\left\langle\mathcal{Q}^{i j}{ }_{j}{ }^{k}\right\rangle & =-\rho\left(A_{1}{ }^{i k}-2 B^{i k}\right), \\
\left\langle\mathcal{Q}^{[i j}{ }_{k}{ }^{l]}\right\rangle & =-\frac{1}{3} \rho\left(A_{2 k}{ }^{l i j}+2 \delta_{k}{ }^{[i} B^{j l]}\right),
\end{aligned}
$$

for the trace and the total antisymmetrization of $\mathcal{Q}_{\underline{A}} i^{j}$ from (2.14).

With this, we can turn to the Scherk-Schwarz reduction of the fermionic sector. The Scherk-Schwarz ansatz for the fermions is simply governed by their respective density weights,

$$
\psi_{\mu}{ }^{i}(x, Y)=\rho^{-\frac{1}{2}}(Y) \psi_{\mu}{ }^{i}(x), \quad \chi^{i j k}(x, Y)=\rho^{\frac{1}{2}}(Y) \chi^{i j k}(x),
$$


in accordance with their behavior under generalized diffeomorphisms. ${ }^{3}$ Indeed, we will confirm in the following that this ansatz reproduces precisely the supersymmetry variations of gauged supergravity. Consider first the gravitino variation in (2.33),

$$
\left\langle\delta_{\epsilon} \psi_{\mu}{ }^{i}\right\rangle=\left\langle 2 \mathcal{D}_{\mu} \epsilon^{i}\right\rangle-4\left\langle\widehat{\nabla}^{i j}\left(\gamma_{\mu} \epsilon_{j}\right)\right\rangle \text {. }
$$

By the results of the previous section the first term reduces to the correct gauge covariantized external derivative $\mathcal{D}_{\mu} \epsilon^{i}$ of gauged supergravity. For the second term using that $\gamma_{\mu} \epsilon_{j}$ has weight $\frac{3}{4}$ the covariant derivative above reads

$$
\begin{aligned}
\widehat{\nabla}^{i j}\left(\gamma_{\mu} \epsilon_{j}\right)= & \left(\mathcal{V}^{-1}\right)^{M i j}\left(e^{\frac{3}{8}} \partial_{M}\left(e^{-\frac{3}{8}} \gamma_{\mu} \epsilon_{j}\right)+\frac{1}{4} e^{\mu \alpha} \partial_{M} e_{\mu}{ }^{\beta} \gamma_{\alpha \beta} \gamma_{\mu} \epsilon_{j}\right)+\frac{1}{2} Q^{i j}{ }_{j}{ }^{k} \gamma_{\mu} \epsilon_{k} \\
& -\frac{1}{16} \mathcal{F}_{\nu \rho}{ }^{i j} \gamma^{\nu \rho} \gamma_{\mu} \epsilon_{j}
\end{aligned}
$$

with the flattened field strength from (2.7). Upon inserting the Scherk-Schwarz ansatz the terms in the first parenthesis are actually $Y$-independent, so this derivative vanishes. Also the second term is zero because under Scherk-Schwarz reduction the first factor is proportional to $\eta^{\alpha \beta}$, which vanishes upon contraction with $\gamma_{\alpha \beta}$. Together, we find with (3.24)

$$
\left\langle\delta_{\epsilon} \psi_{\mu}{ }^{i}\right\rangle=\rho^{-1 / 2}\left(2 \mathcal{D}_{\mu} \epsilon^{i}+\frac{1}{4} \mathcal{F}_{\nu \rho}{ }^{i j} \gamma^{\nu \rho} \gamma_{\mu} \epsilon_{j}+2\left(A_{1}^{i j}-2 B^{i j}\right) \gamma_{\mu} \epsilon_{j}\right)
$$

These are precisely the gravitino variations of gauged supergravity including trombone gaugings, as given in [52] (taking into account the change of normalization explained in footnote 2). In complete analogy, we obtain with the second relation from (3.24)

$$
\left\langle\delta_{\epsilon} \chi^{i j k}\right\rangle=\rho^{1 / 2}\left(-2 \sqrt{2} \mathcal{P}_{\mu}{ }^{i j k l} \gamma^{\mu} \epsilon_{l}+\frac{3 \sqrt{2}}{4} \mathcal{F}_{\mu \nu}{ }^{[i j} \gamma^{\mu \nu} \epsilon^{k]}-2 \sqrt{2} A_{2 l} l^{i j k} \epsilon^{l}-4 \sqrt{2} B^{[i j} \epsilon^{k]}\right) .
$$

Again, this is precisely the expected result for the fermion supersymmetry variation in gauged supergravity including trombone gauging.

Finally, we turn to the Scherk-Schwarz reduction of the scalar potential given in (2.30), with the generalized scalar curvature defined implicitly by (2.28). Upon Scherk-Schwarz reduction the latter equation reads

$$
\frac{1}{16}\left\langle\mathcal{R} \epsilon_{i}\right\rangle=6\left\langle\nabla_{i k} \nabla^{k j} \epsilon_{j}\right\rangle+3\left\langle\nabla^{k j} \nabla_{[i k} \epsilon_{j]}\right\rangle
$$

since $\left\langle\nabla_{M} g_{\mu \nu}\right\rangle=0$. It is then straightforward to determine the Scherk-Schwarz reduction of the various terms in the potential by successive action of (3.23). For instance, the first term reads

$$
\left\langle\nabla_{i k} \nabla^{k j} \epsilon_{j}\right\rangle=\left\langle e^{-\frac{1}{8}} \partial_{i k}\left(e^{\frac{1}{8}} \nabla^{k j} \epsilon_{j}\right)-\frac{1}{2} Q_{i k l}{ }^{k} \nabla^{l j} \epsilon_{j}\right\rangle=-\frac{1}{2}\left\langle Q_{i k l}{ }^{k}\right\rangle\left\langle\nabla^{l j} \epsilon_{j}\right\rangle,
$$

\footnotetext{
${ }^{3}$ We note, in particular, that the ansatz does not carry the Killing spinors of the internal manifold as one might have expected in analogy to standard Kaluza-Klein compactifications. This appears natural, since for the general class of reductions to be discussed the internal space may not even possess sufficiently many Killing spinors. With respect to the supersymmetric reduction ansatz of [6] for the $S^{7}$ case this corresponds to a different $\mathrm{SU}(8)$ gauge choice.
} 
where the vanishing of the first term in here follows from $\nabla \epsilon$ having weight $-\frac{1}{4}$, for which all $Y$-dependence cancels. Similarly, working out the inner covariant derivative we find

$$
\left\langle\nabla_{i k} \nabla^{k j} \epsilon_{j}\right\rangle=-\frac{1}{4}\left\langle Q_{i k l}{ }^{k}\right\rangle\left\langle Q^{l j}{ }_{j}^{k}\right\rangle\left\langle\epsilon_{k}\right\rangle
$$

which by use of (3.24) can be expressed in terms of the gauged supergravity quantities $A_{1}, A_{2}$ and $B$. Performing the same computation for the final two terms in (3.30) we obtain eventually

$$
\begin{aligned}
\frac{1}{16}\left\langle\mathcal{R} \epsilon_{i}\right\rangle= & \rho^{3 / 2}\left(\frac{3}{2} A_{i m} A^{j m}-\frac{1}{12} A_{i}{ }^{k m n} A^{j}{ }_{k m n}+3 A_{i m} B^{j m}-3 B_{i m} A^{j m}\right. \\
& \left.\quad-\frac{1}{6} A_{i}{ }^{j m n} A_{m n}+\frac{5}{6} A^{j}{ }_{i m n} B^{m n}-\frac{64}{9} B_{i m} B^{j m}+\frac{5}{9} B_{m n} B^{m n} \delta_{i}^{j}\right) \epsilon_{j} \\
= & \rho^{3 / 2}\left(\frac{3}{16} A_{m n} A^{m n}-\frac{1}{96} A_{l}{ }^{k m n} A_{k m n}^{l}-\frac{1}{3} B_{m n} B^{m n}\right) \epsilon_{i},
\end{aligned}
$$

where we have used in the last equation the quadratic constraints satisfied by the embedding tensor, c.f. (5.3)-(5.5) of [52]. ${ }^{4}$ This gives precisely the correct scalar potential of gauged supergravity (or, more precisely, the correct contribution to the Einstein field equations in the presence of trombone gauging $B_{i j} \neq 0$ ). An equivalent calculation for the scalar potential has been done in [36] via the generalized Ricci tensor of [42] with the full match to gauged supergravity in absence of the trombone parameter.

To summarize, we have shown that the generalized Scherk-Schwarz ansatz is consistent in the full exceptional field theory and exactly reproduces all field equations and transformation rules of the lower-dimensional gauged supergravity, provided the twist matrices satisfy (3.2) and the section condition (2.1).

\section{Sphere and hyperboloid compactifications}

In this section we construct explicit solutions to the twist equations (3.10) within the SL(5), $\mathrm{E}_{6(6)}$, and $\mathrm{E}_{7(7)}$ exceptional field theories. The twist matrices live within a maximal $\mathrm{SL}(n)$ subgroup (for $n=5, n=6$, and $n=8$, respectively), and describe consistent truncations to lower-dimensional theories with gauge group $\operatorname{CSO}(p, q, r)$ for $p+q+r=n$. For $r=0$, the corresponding internal spaces are warped hyperboloids and spheres, for $r>0$ they also include factors of warped tori.

\section{1 $\mathrm{SL}(n)$ twist equations}

To begin with, let us study the case of the $D=7$, SL(5) theory. As it turns out, this case already exhibits all the structures relevant for the general sphere and hyperboloid compactifications. Although the full SL(5) EFT(including $D=7$ metric, vector, and $p$ form fields) has not yet been constructed, the internal (scalar) sector has been studied in

\footnotetext{
${ }^{4}$ We recall that these constraints are automatically satisfied as a consequence of the section constraints (2.1).
} 
some detail $[34,40,42,60]$. In this case, the underlying group is $\mathrm{SL}(5)$ and vector fields $\mathcal{A}_{\mu}{ }^{A B}$ and internal coordinates $Y^{A B}$ transform in the antisymmetric 10 representation, i.e.

$$
\mathcal{A}_{\mu}^{A B}=\mathcal{A}_{\mu}^{[A B]}, \quad Y^{A B}=Y^{[A B]}, \quad A, B=0, \ldots, n-1,
$$

with $n=5$. In order to prepare the ground for the general case, we will in the following keep $n$ arbitrary and only in the final formulas specify to $n=5 .{ }^{5}$ The section conditions in this case take the $\mathrm{SL}(n)$ covariant form

$$
\partial_{[A B} \otimes \partial_{C D]} \equiv 0
$$

The reduction ansatz for the vector field is given by (3.5)

$$
\mathcal{A}_{\mu}{ }^{A B}(x, Y)=\rho^{-1}\left(U^{-1}\right)_{C D}{ }^{A B} A_{\mu}{ }^{C D}(x)=\rho^{-1}\left(U^{-1}\right)_{C}{ }^{A}\left(U^{-1}\right)_{D}{ }^{B} A_{\mu}{ }^{C D}(x),
$$

in terms of an $\operatorname{SL}(n)$-valued $n \times n$ twist matrix $U_{A}{ }^{B}$. For $D=7$ maximal supergravity, the embedding tensor resides in the $[2,0,0,0] \oplus[0,0,1,1]$ representation [61]

$$
\begin{aligned}
\Theta_{A B, C}{ }^{D}= & \delta_{[A}^{D} \eta_{B] C}+Z_{A B C}{ }^{D}, \\
& \text { with } \eta_{A B}=\eta_{(A B)}, \quad Z_{A B C}{ }^{D}=Z_{[A B C]}{ }^{D}, \quad Z_{A B C}{ }^{C}=0 .
\end{aligned}
$$

Accordingly, the twist equations (3.10) take the form

$$
\begin{aligned}
{\left[\mathcal{X}_{A B, C}\right]_{(\mathbb{P})} } & \stackrel{!}{=} \rho\left(\delta_{[A}^{D} \eta_{B] C}+Z_{A B C}{ }^{D}\right), \\
\partial_{C D}\left(U^{-1}\right)_{A B} C D-(D-1) \rho^{-1} \partial_{C D} \rho\left(U^{-1}\right)_{A B} C D & \stackrel{!}{=} \rho(D-2) \vartheta_{A B}
\end{aligned}
$$

with $D=7$, which for the purpose of later generalisations we also keep arbitrary for the moment and only specify in the final formulas. Here, $\mathcal{X}_{A B, C}{ }^{D}$ denotes the $\operatorname{SL}(n)$ version of $(3.12)$

$$
\mathcal{X}_{A B, C}{ }^{D} \equiv\left(U^{-1}\right)_{A B}^{G H}\left(U^{-1}\right)_{C}^{E} \partial_{G H} U_{E}^{D},
$$

and the projection $[\cdot]_{(\mathbb{P})}$ refers to the projection onto the representations of $\eta_{A B}$ and $Z_{A B C}{ }^{D}$ from (4.4). We can thus write the first equation of (4.5) more explicitly as

$$
\begin{array}{r}
\partial_{C D}\left(U^{-1}\right)_{(A}{ }^{C}\left(U^{-1}\right)_{B)} D \stackrel{!}{=} \frac{1}{2}(1-n) \rho \eta_{A B}, \\
\left(U^{-1}\right)_{A B C}{ }^{G H E} \partial_{G H} U_{E}^{D}-\frac{1}{(n-2)} \partial_{G H}\left(U^{-1}\right)_{[A B}{ }^{G H} \delta_{C]}{ }^{D} \stackrel{!}{=} \rho Z_{A B C}{ }^{D} .
\end{array}
$$

For later use, let us record that in terms of irreducible $\mathrm{SL}(n)$ representations (for general value of $n$ ) the consistency equations (4.5), (4.7) constrain the

$$
[0,1,0, \ldots, 0] \oplus[2,0,0, \ldots, 0] \oplus[0,0,1, \ldots, 0,1],
$$

\footnotetext{
${ }^{5}$ It is in this 'SL $(n)$ generalized geometry' that the generalized parallelizability of spheres has been discussed in [38].
} 
part of the $\mathcal{X}_{A B, C}{ }^{D}$, but leave its

$$
[1,1,0, \ldots, 0,1]
$$

part unconstrained. For the present case $n=5$, this translates into a constrained part $\mathbf{1 0} \oplus \mathbf{1 5} \oplus \mathbf{4 0}^{\prime}$ and an unconstrained $\mathbf{1 7 5}$.

In the following we will construct the twist matrices corresponding to sphere and hyperboloid compactifications. These satisfy the additional conditions

$$
Z_{A B C}{ }^{D}=0, \quad \vartheta_{A B}=0 .
$$

This is consistent with the fact that the resulting gauged supergravities are described by an embedding tensor $\eta_{A B}$ and no trombone symmetry is excited in these compactifications. The consistency equations (4.5), (4.7) thus take the stronger form

$$
\begin{aligned}
& \partial_{C D}\left(U^{-1}\right)_{(A}^{C}\left(U^{-1}\right)_{B)} D \stackrel{!}{=} \frac{1}{2}(1-n) \rho \eta_{A B}, \\
& \partial_{C D}\left[\rho^{1-D}\left(U^{-1}\right)_{A B} C D\right] \stackrel{!}{=} 0, \\
& \left(U^{-1}\right)_{A B C}{ }^{G H E} \partial_{G H} U_{E} D \stackrel{!}{=} \frac{D-1}{n-2}\left(U^{-1}\right)_{[A B}{ }^{G H} \delta_{C]}{ }^{D} \rho^{-1} \partial_{G H} \rho .
\end{aligned}
$$

In the following, we will construct solutions to these equations for arbitrary constant $\eta_{A B}$. Let us note that the $\mathrm{SL}(n)$ covariance of these consistency equations allows to bring $\eta_{A B}$ into diagonal form

$$
\eta_{A B}=\operatorname{diag}(\underbrace{1, \ldots}_{p} \underbrace{-1, \ldots}_{q} \underbrace{0, \ldots}_{r}), \quad \text { with } p+q+r=n
$$

upon conjugation of $U$ and constant $\mathrm{SL}(n)$ rotation of the internal coordinates. The resulting reduced theories are gauged supergravities with gauge group $\operatorname{CSO}(p, q, r)$, defined as the $\mathrm{SL}(n)$ subgroup preserving (4.12). For $r=0$, this is the non-compact semisimple group $\mathrm{SO}(p, q)$, for $r>0$ it corresponds to the non-semisimple group with algebra spanned by matrices $T_{A B}$ :

$$
\left(T_{A B}\right)_{C}^{D} \equiv \eta_{C[A} \delta_{B]}^{D}
$$

\subsection{Sphere and hyperboloid solutions}

Recall that the twist matrices $U_{A}^{B}$ are not only subject to the consistency conditions (4.11a)-(4.11c), but also to the section conditions (4.2). In order to solve the latter, we make the following ansatz

$$
\partial_{i j} U_{A}^{B}=0, \quad \text { for } \quad i, j=1, \ldots, n-1,
$$

i.e. we restrict the coordinate dependence of $U_{A}{ }^{B}$ to the $(n-1)$ coordinates $y^{i} \equiv Y^{0 i}$. For the SL(5) theory this corresponds to restricting the internal part of the exceptional spacetime from 10 to the 4 coordinates that extend the $D=7$ theory to eleven-dimensional 
supergravity [40]. After this reduction, the first of the twist equations (4.11a) splits into the pair of equations

$$
\begin{aligned}
& \partial_{i}\left(U^{-1}\right)_{0}^{0}\left(U^{-1}\right)_{m}^{i}-\partial_{i}\left(U^{-1}\right)_{m}^{i}\left(U^{-1}\right)_{0}^{0} \\
& =\partial_{i}\left(U^{-1}\right)_{0}{ }^{i}\left(U^{-1}\right)_{m}{ }^{0}-\partial_{i}\left(U^{-1}\right)_{m}{ }^{0}\left(U^{-1}\right)_{0}{ }^{i}, \\
& \partial_{i}\left(U^{-1}\right)_{(m}{ }^{0}\left(U^{-1}\right)_{n)}{ }^{i}-\partial_{i}\left(U^{-1}\right)_{\left(m^{i}\right.}{ }^{i}\left(U^{-1}\right)_{n)}{ }^{0} \\
& =\left(\partial_{i}\left(U^{-1}\right)_{0}^{0}\left(U^{-1}\right)_{0}^{i}-\partial_{i}\left(U^{-1}\right)_{0}^{i}\left(U^{-1}\right)_{0}^{0}\right) \eta_{m n},
\end{aligned}
$$

while the density factor $\rho$ is obtained from the $(A B)=(00)$ component as

$$
\rho=\frac{1-n}{2}\left(\partial_{i}\left(U^{-1}\right)_{0}^{0}\left(U^{-1}\right)_{0}^{i}-\partial_{i}\left(U^{-1}\right)_{0}^{i}\left(U^{-1}\right)_{0}^{0}\right) .
$$

Here $\eta_{m n}$ is the reduction of $\eta_{A B}(4.12)$ to the last $n-1$ coordinates, i.e. the diagonal matrix

$$
\eta_{m n}=\operatorname{diag}(\underbrace{1, \ldots}_{p-1} \underbrace{-1, \ldots}_{q} \underbrace{0, \ldots}_{r}) .
$$

We will first treat the case $r=0$ of non-degenerate $\eta_{m n}$ and subsequently extend the discussion to the general case.

\subsubsection{The case $\mathrm{SO}(p, q)$}

For $\eta_{m n}$ given by (4.17) with $r=0$, equations (4.15) can be solved by the following explicit $\mathrm{SL}(n)$ ansatz

$$
\begin{aligned}
\left(U^{-1}\right)_{0}{ }^{0} & \equiv(1-v)^{(n-1) / n}, \\
\left(U^{-1}\right)_{0}{ }^{i} & \equiv \eta_{i j} y^{j}(1-v)^{(n-2) /(2 n)} K(u, v), \\
\left(U^{-1}\right)_{i}{ }^{0} & \equiv \eta_{i j} y^{j}(1-v)^{(n-2) /(2 n)}, \\
\left(U^{-1}\right)_{i}{ }^{j} & \equiv(1-v)^{-1 / n}\left(\delta^{i j}+\eta_{i k} \eta_{j l} y^{k} y^{l} K(u, v)\right),
\end{aligned}
$$

with $n=p+q$ and the combinations $u \equiv y^{i} y^{i}, v \equiv y^{i} \eta_{i j} y^{j}$. Upon inserting this ansatz into (4.15), these equations reduce to a single differential equation for the function $K(u, v)$, given by

$$
2(1-v)\left(u \partial_{v} K+v \partial_{u} K\right)=((1+q-p)(1-v)-u) K-1
$$

This equation takes a slightly simpler form upon change of variables

$$
u \equiv r^{2} \cosh \varphi, \quad v \equiv r^{2} \sinh \varphi,
$$

after which it becomes an ordinary differential equation in $\varphi$

$$
2\left(1-r^{2} \sinh \varphi\right) \partial_{\varphi} K=\left((1+q-p)\left(1-r^{2} \sinh \varphi\right)-r^{2} \cosh \varphi\right) K-1 .
$$

This can be solved analytically for any pair of integers $(p, q)$. We have to treat separately the cases $q=0$ and $p=1$ (corresponding to $\mathrm{SO}(p)$ and $\mathrm{SO}(1, q)$ gaugings, respectively) for 
which $u= \pm v$ and the change of variables (4.20) does not make sense. In the former case $u=v$, and equation (4.19) reduces to

$$
2 u(1-u) K^{\prime}=(1-p+p u-2 u) K-1,
$$

with the particular solution

$$
K=-{ }_{2} F_{1}[1,(p-2) / 2 ; 1 / 2 ; 1-u],
$$

in terms of the hypergeometric function ${ }_{2} F_{1}$. Similarly, for $p=1$, we have $u=-v$, and equation (4.19) reduces to

$$
-2 u(1+u) K^{\prime}=(q+q u-u) K-1,
$$

with particular solution

$$
K=q^{-1}\left(1+u(1-q){ }_{2} F_{1}[1,(1+q) / 2 ; 1 / 2 ; 1+u]\right) .
$$

Finally, the density factor $\rho$ can be read off from (4.16) as

$$
\rho=\rho_{p, q} \equiv(1-v)^{(n-4) /(2 n)} .
$$

We have thus fully determined the twist matrix $U$ and the density factor $\rho$ and entirely solved the first of the twist equations (4.11a). It remains to verify the other two equations.

With the twist matrix given by (4.18) and using the differential equation (4.19) for the function $K$, it is straightforward to verify that

$$
\partial_{i}\left[\left(U^{-1}\right)_{[A}^{0}\left(U^{-1}\right)_{B]}^{i}\right]=\frac{n-2}{n} \frac{2 \eta_{i j} y^{j}}{1-v}\left(U^{-1}\right)_{[A}^{0}\left(U^{-1}\right)_{B]}{ }^{i} .
$$

Together with the form of the density factor $\rho$ from (4.26), we thus find, that the second equation $(4.11 \mathrm{~b})$ is identically satisfied provided we have the relation

$$
\frac{1}{2}(D-1)=\frac{n-2}{n-4},
$$

relating the number of external space-time dimensions to the size of the group $\operatorname{SL}(n)$. Fortunately, this relation holds precisely in the case $D=7, n=5$ that we are interested in. We have thus shown that also the second twist equation (4.11b) holds for our choice of twist matrix and density factor. Let us note that integer solutions of equation (4.28) are very rare and essentially restrict to

$$
(D, n)=(7,5), \quad(D, n)=(5,6), \quad(D, n)=(4,8),
$$

in which we recover the dimensions of the known sphere compactifications $\operatorname{AdS}_{D} \times \mathrm{S}^{n-1}$ of eleven-dimensional and type IIB supergravity. We will come back to this in section 4.3. As a last consistency check, one verifies by direct computation that the last twist equation (4.11c) is also identically satisfied for (4.18) with (4.19). This essentially follows from the fact that no object fully antisymmetric in three indices $[A B C]$ can be constructed from the explicit $\eta_{i j} y^{j}$.

To summarize, we have shown that the $\mathrm{SL}(n)$ twist matrix $U$ given by (4.18) with (4.19), together with the density factor (4.26) satisfies the consistency equations (4.11a)-(4.11c), provided the integer relation (4.28) holds. In the next section, we will generalize this solution to include the case $r>0$. 


\subsubsection{The case $\operatorname{CSO}(p, q, r)$}

The solution of equations equations (4.11a)-(4.11c) derived in the previous section can be generalized to the case $r>0$, in which the reduced theory comes with the gauge group $\operatorname{CSO}(p, q, r)$. A natural ansatz for the $\operatorname{SL}(p+q+r)$ twist matrix in this case is given by

$$
\left(U^{-1}\right)_{A}{ }^{B}=\left(\begin{array}{c:c}
\beta^{-r} U_{(p, q)}^{-1} & 0 \\
\hdashline 0 & \beta^{p+q} \mathbb{I}_{r}
\end{array}\right),
$$

where $U_{(p, q)}^{-1}$ denotes the $\mathrm{SO}(p, q)$ solution from (4.18), (4.19), and $\mathbb{I}_{r}$ is the $r \times r$ identity matrix. The factor $\beta=\beta(v)$ is a function of $v \equiv y^{i} \eta_{i j} y^{j}$ and put such that the determinant of the twist matrix remains equal to one. Note that the twist matrix only depends on coordinates $y^{i}, i=1, \ldots, p+q-1$.

Let us now work out equations (4.11a)-(4.11c) for the ansatz (4.30). The first equation (4.11a) is solved identically by this ansatz without any assumption on the function $\beta$, as a mere consequence of the fact that $U_{(p, q)}$ solves the corresponding equations for $\mathrm{SO}(p, q)$. Indeed, all components of this equation in which one of the free indices $(A B)$ takes values beyond $p+q-1$, hold trivially due to the block-diagonal structure of the twist matrix (4.30) and the fact that the matrix does not depend on the last $r$ coordinates. The constraint equations then simply reduce to their $\mathrm{SO}(p, q)$ analogues. The presence of the factor $\beta^{-r}$ does not spoil the validity of the equation, but contributes to the density factor $\rho$ as

$$
\rho=\rho_{p, q, r} \equiv \beta^{-2 r} \rho_{p, q},
$$

with $\rho_{p, q}$ from (4.26). We continue with the second equation (4.11b) which now takes the form

$$
\partial_{C D}\left[\rho_{p, q}^{1-D} \beta^{2 r(D-2)}\left(U_{(p, q)}^{-1}\right)_{A B} C D\right] \stackrel{!}{=} 0,
$$

and thus reduces to the identity (4.27) provided we choose $\beta$ such that

$$
\rho_{p, q}^{1-D} \beta^{2 r(D-2)}=(1-v)^{-(p+q-2) /(p+q)} .
$$

With (4.26), this reduces to

$$
\beta^{r}=(1-v)^{(D-3) /(4(D-2))-1 /(p+q)} .
$$

Even though the $\operatorname{CSO}(p, q, r)$ case seems to admit more freedom in that we are not bound by a relation (4.28) to fix the size of the external space-time, we will for the moment restrict to the three principal cases (4.29), i.e. keep the additional relation (4.28)

$$
\frac{1}{2}(D-1)=\frac{p+q+r-2}{p+q+r-4},
$$

and describe reductions to four, five and seven dimensions, respectively. Then, the form of the scale factor $\beta$ from (4.34) simplifies to

$$
\beta=(1-v)^{-1 /((p+q)(p+q+r))} .
$$


Together with (4.30) this fully defines the twist matrix that solves the consistency equations (4.11a)-(4.11c) for $\eta_{A B}$ from (4.12). As above, the last equation (4.11c) is verified by explicit calculation.

To summarize, we have shown that the $\operatorname{SL}(p+q+r)$ twist matrix $U_{M}{ }^{N}$ given by (4.18), (4.30), (4.36), together with the density factor (4.31), satisfies the consistency equations (4.11a)-(4.11c) for the general $\eta_{A B}$ of (4.12), provided the integer relation (4.35) holds. By the above discussion, this applies in particular to the case $D=7, \mathrm{G}=\mathrm{SL}(5)$, and implies that the resulting twist ansatz describes a consistent truncation of the corresponding EFT. Since this twist matrix also falls into the class (4.14) of solutions to the section conditions, this generalized Scherk-Schwarz ansatz describes consistent truncations of the full $D=11$ supergravity down to seven-dimensional supergravities with gauge groups $\mathrm{CSO}(p, q, r),(p+q+r=5)$. We will work out in section 4.4 the internal background metrics induced by these twist matrices, in order to get the geometrical perspective for these compactifications.

\section{$4.3 \quad \mathrm{E}_{6(6)}$ and $\mathrm{E}_{7(7)}$ twist equations}

In this section, we show that the $\mathrm{SL}(n)$ twist matrices found in the previous section can also be used for the construction of solutions to the consistency equations (3.10) in the exceptional field theories with groups $\mathrm{E}_{6(6)}$ and $\mathrm{E}_{7(7)}$. The structure underlying this construction is the embedding of the $\mathrm{SL}(n)$ twist matrices via

$$
\mathrm{SL}(6) \subset \mathrm{E}_{6(6)}, \quad \mathrm{SL}(8) \subset \mathrm{E}_{7(7)}
$$

respectively, inducing a decomposition of the $\mathrm{E}_{n(n)}$ coordinates according to

$$
\begin{gathered}
Y^{M} \longrightarrow\left\{Y^{[A B]}, \ldots\right\} \longrightarrow\left\{y^{i}, \ldots\right\}, \quad \text { with } y^{i} \equiv Y^{[0 i]} \\
A, B=0, \ldots, n-1, \quad i=1, \ldots, n-1
\end{gathered}
$$

Together with a solution of the section constraint achieved by restricting the coordinate dependence of all fields according to

$$
\Phi\left(Y^{M}\right) \longrightarrow \Phi\left(y^{i}\right)
$$

and the fact that both cases (4.37) correspond to a solution of the integer relation (4.28) (with $(D, n)=(5,6)$ and $(D, n)=(4,8)$, respectively), this structure turns out to be sufficient to ensure that the $\mathrm{SL}(n)$ twist matrices constructed above also define solutions to the consistency equations (3.10) of these larger exceptional field theories. The corresponding Scherk-Schwarz ansatz then defines lower-dimensional theories with embedding tensor describing the gauge groups $\mathrm{CSO}(p, q, r),(p+q+r=n)$.

\subsection{1 $\quad \mathrm{E}_{6(6)}$}

For details about the $\mathrm{E}_{6(6)}$ exceptional field theory, we refer to [45]. It is formulated on an internal space of 27 coordinates $Y^{M}$ in the fundamental representation of $\mathrm{E}_{6(6)}$, with the 
section condition given by the 27 equations $d^{K M N} \partial_{M} \otimes \partial_{N} \equiv 0$, with the cubic invariant $d^{K M N}$. In this case, the subgroup $\mathrm{SL}(6)$ is embedded into $\mathrm{E}_{6(6)}$ via

$$
\mathrm{SL}(6) \subset \mathrm{SL}(6) \times \mathrm{SL}(2) \subset \mathrm{E}_{6(6)}
$$

with the internal coordinates decomposing as

$$
\overline{\mathbf{2 7}} \longrightarrow(15,1)+\left(6^{\prime}, 2\right) .
$$

The ten-dimensional IIB theory is recovered from $\mathrm{E}_{6(6)}$ exceptional field theory upon solving the associated section condition by restricting the coordinate dependence of all fields to 5 coordinates within the $(15,1)$ (transforming as a vector under the maximal GL(5) subgroup). Specifically, with (4.41), we decompose coordinates as

$$
Y^{M} \longrightarrow\left\{Y^{[A B]}, Y_{A \alpha}\right\}, \quad \text { with } \quad A=0, \ldots, 5, \quad \alpha=1,2,
$$

and impose

$$
\partial_{i j} \Phi=0, \quad \partial^{0 \alpha} \Phi=0, \quad \partial^{i \alpha} \Phi=0,
$$

for $i=1, \ldots, 5$. Comparing to (4.2), we observe that the $\mathrm{SL}(6)$ twist matrix constructed above is compatible with this solution of the section condition. Upon the embedding (4.40), (4.41), it gives rise to an $\mathrm{E}_{6(6)}$ twist matrix $U_{M}{ }^{N}$

$$
U_{M}^{N}=\left(\begin{array}{c:c}
U_{[A B]}[C D] & 0 \\
\hdashline 0 & \delta_{\gamma}^{\alpha}\left(U^{-1}\right)_{C}^{A}
\end{array}\right),
$$

satisfying (4.43). As a consequence, the generalized Scherk-Schwarz ansatz (3.5) for the full EFT

$$
\begin{aligned}
e_{\mu}{ }^{\alpha}(x, Y) & =\rho^{-1}(Y) e_{\mu}{ }^{\alpha}(x), \\
\mathcal{M}_{M N}(x, Y) & =U_{M}{ }^{P}(Y) U_{N}{ }^{Q}(Y) M_{P Q}(x), \\
\mathcal{A}_{\mu}{ }^{M}(x, Y) & =A_{\mu}{ }^{N}(x)\left(U^{-1}\right)_{N}{ }^{M}(Y) \rho^{-1}(Y), \\
\mathcal{B}_{\mu \nu M}(x, Y) & =\rho^{-2}(Y) U_{M}{ }^{P}(Y) B_{\mu \nu P}(x),
\end{aligned}
$$

describes consistent truncations from IIB supergravity to $D=5$ theories, provided the twist matrix (4.44) solves the full set of consistency conditions (3.10).

Let us thus consider the matrix (4.44) built from the $\mathrm{SL}(6)$ matrix $U_{A}{ }^{B}$ from $(4.30),(4.36)$, which in turn solves equations (4.11a)-(4.11c) for general $\eta_{A B}$ characterised by three integers $p+q+r=6$. The first consistency equation (3.11) for the $\mathrm{E}_{6(6)}$ twist matrix (4.44) reads

$$
\rho^{-1}\left[\mathcal{X}_{M}^{\boldsymbol{\alpha}}\right]_{(\mathbf{3 5 1})} \stackrel{!}{=} \text { const , }
$$

with $\mathcal{X}_{M}{ }^{\alpha}$ defined in (3.12). It follows from the form of the matrix (4.44) together with (4.43) that the only non-vanishing components of $\mathcal{X}_{M}{ }^{\boldsymbol{\alpha}}$ from (3.12) are its components $\mathcal{X}_{[A B]}{ }^{\boldsymbol{\alpha}}$ when $\boldsymbol{\alpha}$ takes values in the $\mathrm{SL}(6)$ subgroup of $\mathrm{E}_{6(6)}$. These are nothing 
but the $\mathcal{X}_{A B, C}{ }^{D}$ defined directly in terms of $U_{A}{ }^{B}$ in (4.6) above, and moreover singlets under the $\mathrm{SL}(2)$ of (4.40). For equation (4.46) this means that under decomposition w.r.t. SL(6) its only non-vanishing components are the SL(2) singlets in the branching of $\mathbf{3 5 1} \longrightarrow(21,1)+(105,1)+\ldots$, reducing to

$$
\rho^{-1}\left[\mathcal{X}_{M}^{\alpha}\right]_{(21)} \stackrel{!}{=} \text { const }, \quad \rho^{-1}\left[\mathcal{X}_{M}^{\alpha}\right]_{(105)} \stackrel{!}{=} \text { const . }
$$

Comparing to the general discussion around (4.8), (4.9), these equations are precisely ensured by the properties of the SL(6) twist matrix $U_{A}{ }^{B}$ constructed above, as a consequence of (4.5). Specifically, we find that

$$
\rho^{-1}\left[\mathcal{X}_{M}^{\boldsymbol{\alpha}}\right]_{(A B)}=\eta_{A B}, \quad \rho^{-1}\left[\mathcal{X}_{M}^{\boldsymbol{\alpha}}\right]_{(105)}=0
$$

and conclude that the first consistency equation in (3.10) is solved by (4.44) with the density factor $\rho$ given by (4.31) above. It remains to study the second equation from (3.10). Again, the structure of the matrix (4.44) and its coordinate dependence (4.43) imply that the l.h.s. of this equation reduces to

$$
\partial_{C D}\left(U^{-1}\right)_{A B}^{C D}-(D-1) \rho^{-1} \partial_{C D} \rho\left(U^{-1}\right)_{A B}^{C D},
$$

which vanishes for the $\mathrm{SL}(6)$ twist matrix $U_{A}{ }^{B}$ due to its property (4.11b), by virtue of the integer relation (4.28), which holds for the present case $(D, n)=(5,6)$.

We conclude, that the twist matrix (4.44) with $U_{A}^{B}$ given by (4.30), (4.36) above, together with the density factor $\rho_{p, q, r}$ from (4.31) satisfies both the section constraints (as a subclass of the general IIB solution (4.43)), and the consistency equations (3.10). Via (4.48) it corresponds to an embedding tensor in the 21 of SL(6) parametrized by the diagonal matrix $\eta_{A B}$ from (4.12). The generalized Scherk-Schwarz ansatz thus describes the consistent truncation from $D=10$ IIB supergravity to a maximal $D=5$ gauged supergravity with gauge group $\operatorname{CSO}(p, q, r)$.

\subsection{2 $\mathrm{E}_{7(7)}$}

This case works in complete analogy to $\mathrm{E}_{6(6)}$. We have reviewed the $\mathrm{E}_{7(7)}$ exceptional field theory in section 2 above. The relevant subgroup is $\mathrm{SL}(8)$ embedded into $\mathrm{E}_{7(7)}$ such that the 56 internal coordinates decompose as

$$
\mathbf{5 6} \longrightarrow 28+28^{\prime}, \quad Y^{M} \longrightarrow\left\{Y^{[A B]}, Y_{[A B]}\right\}
$$

The full $D=11$ theory is recovered from $\mathrm{E}_{7(7)}$ exceptional field theory upon solving the associated section condition by restricting the coordinate dependence of all fields as

$$
\partial_{i j} \Phi=0, \quad \partial^{A B} \Phi=0
$$

for $i=1, \ldots, 7, A=0, \ldots, 7$. Comparing to (4.2), we observe that the $\mathrm{SL}(8)$ twist matrix constructed above is compatible with this solution of the section condition. Upon the embedding (4.50), it gives rise to an $\mathrm{E}_{7(7)}$ twist matrix $U_{M}{ }^{N}$

$$
U_{M}^{N}=\left(\begin{array}{c:c}
U_{[A B]}[C D] & 0 \\
\hdashline 0 & \left(U^{-1}\right)_{[C D]}^{[A B]}
\end{array}\right)
$$


satisfying (4.51). As a consequence, the generalized Scherk-Schwarz ansatz (3.5) for the full EFTdescribes consistent truncations from $D=11$ supergravity to $D=4$ theories, provided the twist matrix (4.52) solves the full set of consistency conditions (3.2).

The first of these equations written in the form (3.11) reads

$$
\rho^{-1}\left[\mathcal{X}_{M}^{\boldsymbol{\alpha}}\right]_{(\mathbf{9 1 2})} \stackrel{!}{=} \text { const }
$$

with $\mathcal{X}_{M}{ }^{\alpha}$ defined in (3.12). It follows from the form of the matrix (4.52) together with (4.51) that the only non-vanishing components of $\mathcal{X}_{M}{ }^{\alpha}$ from (3.12) are its components $\mathcal{X}_{[A B]}{ }^{\alpha}$ when $\boldsymbol{\alpha}$ takes values in the $\mathrm{SL}(8)$ subgroup of $\mathrm{E}_{7(7)}$. As for the $\mathrm{E}_{6(6)}$ case, these are nothing but the $\mathcal{X}_{A B, C}{ }^{D}$ defined directly in terms of $U_{A}{ }^{B}$ in (4.6) above. For equation (4.46) this means that under decomposition w.r.t. SL(8) its only non-vanishing components are given by

$$
\rho^{-1}\left[\mathcal{X}_{M}^{\boldsymbol{\alpha}}\right]_{(36)} \stackrel{!}{=} \text { const }, \quad \rho^{-1}\left[\mathcal{X}_{M}^{\boldsymbol{\alpha}}\right]_{(420)} \stackrel{!}{=} \text { const } .
$$

Comparing to the general discussion around (4.8), (4.9), these equations are precisely ensured by the properties of the SL(8) twist matrix $U_{A}{ }^{B}$ constructed above, as a consequence of (4.5). Specifically, we find that

$$
\rho^{-1}\left[\mathcal{X}_{M}^{\boldsymbol{\alpha}}\right]_{(A B)}=\eta_{A B}, \quad \rho^{-1}\left[\mathcal{X}_{M}^{\boldsymbol{\alpha}}\right]_{(420)}=0
$$

and conclude that the first consistency equation in (3.2) is solved by (4.44) with the density factor $\rho$ given by (4.31) above. It remains to study the second equation. Again, the structure of the matrix (4.52) and its coordinate dependence (4.51) imply that the l.h.s. of this equation reduces to

$$
\partial_{C D}\left(U^{-1}\right)_{A B}^{C D}-3 \rho^{-1} \partial_{C D} \rho\left(U^{-1}\right)_{A B}^{C D},
$$

which vanishes for the SL(8) twist matrix $U_{A}^{B}$ due to its property (4.11b), by virtue of the integer relation (4.28), which holds for the present case $(D, n)=(4,8)$. In full analogy to the $\mathrm{E}_{6(6)}$ case we find that with the ansatz (4.52) for the $\mathrm{E}_{7(7)}$ matrix $U_{M}{ }^{N}$, all nonvanishing parts of the consistency equations (3.10) precisely reduce to the corresponding equations (4.5) for the $\mathrm{SL}(n)$ matrix $U_{A}{ }^{B}$.

We conclude, that the twist matrix (4.52) with $U_{A}^{B}$ given by (4.30), (4.36) above, together with the density factor $\rho_{p, q, r}$ from (4.31) satisfies both the section constraints (as a subclass of the general $D=11$ solution (4.51)), and the consistency equations (3.2). Via (4.55) it corresponds to an embedding tensor in the $\mathbf{3 6}$ of SL(8) parametrized by the diagonal matrix $\eta_{A B}$ from (4.12). The generalized Scherk-Schwarz ansatz thus describes the consistent truncation from $D=11$ supergravity to a maximal $D=4$ gauged supergravity with gauge group $\mathrm{CSO}(p, q, r)$.

\subsection{The induced space-time metric}

In the above, we have constructed twist matrices as solutions of the consistency equations (3.10) which define consistent truncations of the higher-dimensional $D=11$ and 
IIB supergravity down to $D=4,5,7$ maximal supergravity with gauge group $\operatorname{CSO}(p, q, r)$. While consistency of the truncation follows from the general structure of the ansatz and the covariant formulation of exceptional field theory, for physical applications one will typically be interested in the explicit embedding of the lower-dimensional fields into $D=11$ and IIB supergravity in their original form. The translation of the very compact ansatz (3.5) into the original fields of higher-dimensional supergravity thus requires the explicit dictionary between the fields of the exceptional field theory and the original supergravities $[45,46]$.

As an example, let us work out the form of the internal background metric to which the above compactifications correspond. The internal components of the higher-dimensional metric sit among the components of the scalar matrix $\mathcal{M}_{M N}=\left(\mathcal{V V}^{T}\right)_{M N}$, built from a group-valued vielbein $\mathcal{V}$ that carries the higher-dimensional components according to the decomposition of the Lie algebra w.r.t. to a proper grading, c.f. (2.35). See [58] for the general structure of these parametrizations, and [41] for some explicit matrices.

As a general feature of the theories with SL( $n)$ embedding according to (4.38), (4.39), the generalized metric $\mathcal{M}^{M N}$ decomposes into blocks

$$
\mathcal{M}^{M N}=\left(\begin{array}{c:c:c}
\mathcal{M}^{0 i, 0 j} & \mathcal{M}^{0 i, j k} & \cdots \\
\hdashline \mathcal{M}^{i j, 0 k} & \mathcal{M}^{i j, k l} & \cdots \\
\hdashline \vdots & \vdots & \ddots
\end{array}\right)
$$

and the higher-dimensional internal metric $g_{i j}$ can be read off from the upper left block as

$$
\mathcal{M}^{i 0, j 0}=g^{(4-n) / n} g^{i j}
$$

The power of $g$ on the r.h.s. is fixed by the fact that generalized diffeomorphisms on $\mathcal{M}^{M N}$ translate into standard diffeomorphisms for $g^{i j}{ }^{6}{ }^{6}$ For the moment, we are just interested in the higher-dimensional metric at the 'origin' of the truncation, i.e. at the point where all lower-dimensional scalar fields vanish. According to (3.1), at this point, $\mathcal{M}_{M N}$ is given by

$$
\mathcal{M}^{M N}=\left(U^{-1}\right)_{P}^{M}\left(U^{-1}\right)_{P}^{N},
$$

in terms of the twist matrix $U$. Since $U$ is embedded in the subgroup $\operatorname{SL}(n)$, c.f. (4.44), (4.52), the relevant block (4.58) of $\mathcal{M}$ can simply be expressed as

$$
\mathcal{M}^{i 0, j 0}=\frac{1}{2}\left(m^{i j} m^{00}-m^{i 0} m^{j 0}\right)
$$

for $m^{A B} \equiv\left(U^{-1}\right)_{C}^{A}\left(U^{-1}\right)_{C}{ }^{B}$. With the explicit form (4.30) of the twist matrices constructed in section 4.2 for gauge group $\operatorname{CSO}(p, q, r)$, we can thus work out the internal metric, and find after some calculation

$$
\begin{aligned}
d s^{2} & =g_{i j} d y^{i} d y^{j} \\
& =(1+u-v)^{-2 /(p+q+r-2)}\left(d y^{z} d y^{z}+d y^{a} d y^{b}\left(\delta^{a b}+\frac{\eta_{a c} \eta_{b d} y^{c} y^{d}}{1-v}\right)\right)
\end{aligned}
$$

\footnotetext{
${ }^{6} \mathrm{~A}$ short calculation shows compatibility with the explicit result (5.25) of [45] for $\mathrm{E}_{6(6)}$.
} 

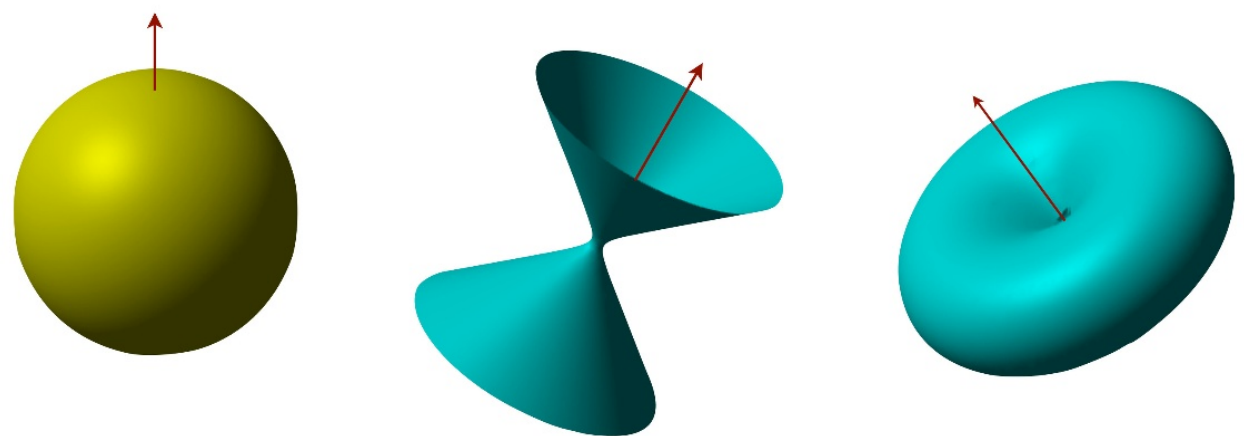

Figure 1. Sphere, hyperboloid and the warped hyperboloid (4.61) in a $2 d$ projection, i.e. for $(p, q, r)=(2,1,0)$.

with the split of coordinates $y^{i}=\left\{y^{a}, y^{u}\right\}, a=1, \ldots, p+q-1$, and $z=p+q, \ldots, r$, and the combinations $u \equiv y^{a} y^{a}, v \equiv y^{a} \eta_{a b} y^{b}$. This space is conform to the direct product

$$
H^{p, q} \times \mathbb{R}^{r},
$$

of $r$ flat directions and the hyperboloid $H^{p, q}$. The latter is defined by the embedding surface

$$
y^{A} \eta_{A B} y^{B}=1, \quad A=0, \ldots, p+q-1,
$$

with $\eta_{A B}$ from (4.12), within a $(p+q)$ dimensional Euclidean space. For $r=q=0$, the metric (4.61) is the round sphere (the pre-factor becomes constant: $1+u-v \rightarrow 1$ ). For $p+q+r=8$, this is precisely the metric proposed by Hull and Warner in [53] with the warp factor deforming the hyperboloid geometry, see figure 1 . This is the higher-dimensional background inducing the $\operatorname{CSO}(p, q, r)$ gauged supergravities in $D=4$ dimensions. Along the very same lines, the higher-dimensional metric can be computed for arbitrary values of the lower-dimensional scalar fields, i.e. for arbitrary values of the matrix $M_{P Q}(x)$, in which case (4.59) is replaced by the full Scherk-Schwarz ansatz (3.1). The uplift of all the $D$-dimensional fields, i.e. all the non-linear reduction ansätze follow straightforwardly (although by somewhat lengthy calculation) from combining this ansatz with the dictionary of the full exceptional field theory to higher-dimensional supergravity, which is independent of the particular form of the twist matrix.

We stress once more, that the metric (4.61) is in general not part of a solution of the higher-dimensional field equations. This simply translates into the fact that the corresponding lower-dimensional supergravity in general does not possess a solution with all scalar fields vanishing. Indeed, it was shown in [53] for the $\mathrm{SO}(p, 8-p)$ supergravities that the metric (4.61) is compatible with a generalized Freund-Rubin ansatz only for the values $(p, q)=(8,0),(p, q)=(4,4)$, and $(p, q)=(5,3)$, which precisely correspond to the gauged supergravities admitting critical points at the origin of the scalar potential. Independently of this property concerning the ground state, in all cases the generalized Scherk-Schwarz ansatz (3.1), (3.5), continues to describe the consistent truncation of the higher-dimensional supergravity to the field content and the dynamics of a lower-dimensional maximally supersymmetric supergravity. 


\section{Conclusions}

In this paper we have shown how the consistency of a large class of Kaluza-Klein truncations can be proved using exceptional field theory. The Kaluza-Klein ansaetze are given in terms of twist matrices $U_{M}{ }^{N}$ taking values in the duality group $\mathrm{E}_{d(d)}$ subject to the consistency equations (3.10). The resulting effective gauged supergravities emerge naturally in the embedding tensor formalism, upon identifying the embedding tensor with a particular projection of $U^{-1} \partial U$. Such generalized Scherk-Schwarz reductions have been considered before in various studies for truncations of the full exceptional field theory. Here, we have given the Scherk-Schwarz ansatz for the full field content of exceptional field theory, including fermions, $p$-forms, and the constrained compensating tensor gauge fields. We have then shown that the ansatz reproduces the complete field equations of the lower-dimensional gauged supergravity. This is necessary in order to relate to the full untruncated $D=11$ or type IIB supergravity. Secondly, we explicitly constructed the twist matrices for various compactifications, including sphere compactifications such as $\mathrm{AdS}_{5} \times \mathrm{S}^{5}$, and new examples such as hyperboloids $H^{p, q}$. In contrast to ordinary Scherk-Schwarz compactifications where the existence of consistent twist matrices is guaranteed by the underlying Lie algebraic structure of the deformation parameters, to our knowledge there is no analogue of such an existence proof for solutions of the generalized consistency equations (3.10) with general embedding tensor $\Theta_{M}{ }^{\alpha}$. In this regard, the construction of explicit examples is a crucial step towards a more systematic understanding of the underlying structures.

Given the explicit form of the twist matrices, any solution of gauged supergravity is embedded into the higher-dimensional exceptional field theory via the relations (1.1)(1.4), and thereby further into $D=11$ or type IIB supergravity. The explicit embedding formulas into the original $D=11$ or type IIB supergravity require the dictionary relating the exceptional field theory fields to the original formulation of these theories. It should be stressed that this dictionary is completely independent of the particular compactification or twist matrix and can be fixed, for instance, by matching the gauge symmetries on both sides. Together this allows in particular to lift the known AdS solutions [62] of $\mathrm{SO}(8)$ gauged supergravity to eleven dimensions, but also the large class of dS and domain wall solutions found in the non compact and non-semisimple four-dimensional gaugings [63-65].

It will be interesting to explore the possible generalizations of the presented construction. Our construction of twist matrices was based on the maximal embedding (4.37), (4.38) of an $\mathrm{SL}(n)$ group into the corresponding exceptional group. For the $\mathrm{SO}(p, q)$ case, as a consequence of the second equation in (3.10), this led to the severe constraint (4.28), restricting the construction to the three principal cases $(D, n)=(7,5),(5,6),(4,8)$. In the $\operatorname{CSO}(p, q, r)$ case on the other hand, the structure of the twist matrix (4.30) suggests that there is more freedom due to the possible introduction of the factor $\beta$, preserving the $\operatorname{CSO}(p, q, r)$ structure. As a result, the construction will still go through without being constrained to (4.35), i.e. in particular for other values of $D$, as given in (4.34). This will be interesting to explore. Similarly, the construction should allow for more solutions, when the embedding (4.38) of $\mathrm{SL}(n)$ is not maximal but leaves additional abelian factors. This is the case for the $D=6, \mathrm{G}=\mathrm{SO}(5,5)$ EFTwith $\mathrm{SL}(5)$ embedded via the intermediate GL(5). The additional GL(1) factor allows for an additional parameter in the twist matrix, 
that can be tuned such as to solve the second equation in (3.10) without reverting to (4.28). This case should thus include the consistent $S^{4}$ reduction of IIA supergravity [66].

A different class of twist matrices should correspond to solutions of (4.5) in which (4.10) is relaxed to non-vanishing $Z_{A B C}{ }^{D}$. E.g. $D=7$ maximal supergravity possesses a gauging with $\eta_{A B}=0$ and compact $\mathrm{SO}(4)$ gauge group [61] which is conjectured to correspond to a consistent sphere truncation of IIB supergravity. It would be interesting to work out the corresponding twist matrix and to explore its generalization to arbitrary values of $n$. It should of course also be possible to find twist matrices that generate a non-vanishing trombone parameter $\vartheta_{M}$. More generally, one may try to generalize the above construction by replacing (4.38) to an embedding of coordinates via other classes of subgroups. The method should also extend to non-maximal theories such as the $\mathrm{AdS}_{3} \times S^{3}$ reduction from six dimensions $[67,68]$.

Another extension of our results that may eventually become feasible is the inclusion of higher-derivative $\alpha^{\prime}$ or M-theory corrections. Indeed, in double field theory there was progress recently of how to include $\alpha^{\prime}$ corrections [69-72], see also [73-75]. In particular, the results of [69] provide an exactly $\mathrm{O}(d, d)$ invariant description of a subsector of heterotic string theory to all orders in $\alpha^{\prime}$. If a generalization to exceptional field theory exists one may hope for consistent Kaluza-Klein embeddings that not only lead to exact solutions of the higher-dimensional field equations but also to solutions that are exact in $\alpha^{\prime}$.

Finally, let us stress that throughout this paper we assumed the strong form of the section constraints (2.1). Thereby the twist matrices we construct as solutions of (3.10) describe consistent truncations within the original $D=11$ and IIB supergravity. It is intriguing, however, that the match with lower-dimensional gauged supergravity, upon reduction by the Scherk-Schwarz ansatz, does not explicitly use the section constraint (provided the initial scalar potential is written in an appropriate form), as observed in [34, 36] for the internal sector and shown here for the full theory. Formally this allows to reproduce all gauged supergravities, and it is intriguing to speculate about their higherdimensional embedding upon possible relaxation of the section constraints that would define a genuine extension of the original supergravity theories. For the moment it is probably fair to say that our understanding of a consistent extension of the framework is still limited. In this context it would be interesting to obtain explicit examples of twist matrices that satisfy all consistency conditions (3.10), but violate the section constraints (2.1) which may give a hint as to how to consistently relax these constraints in exceptional field theory. We hope that our treatment of sphere and hyperboloid compactifications may help clarify these matters.

\section{Acknowledgments}

We would like to thank Hadi and Mahdi Godazgar, Falk Hassler, Dieter Lüst, Diego Marques and Mario Trigiante for interesting discussions for interesting discussions. This work is supported by the U.S. Department of Energy (DoE) under the cooperative research agreement DE-FG02-05ER41360. The work of O.H. is supported by a DFG Heisenberg fellowship. 


\section{A Projection of the spin connection}

Consider a general tensor $W_{M N}{ }^{K}=W_{M}{ }^{\alpha}\left(t_{\boldsymbol{\alpha}}\right)_{N}{ }^{K}$, living in the full tensor product

$$
56 \otimes 133=56 \oplus 912 \oplus 6480 .
$$

In flattened $\mathrm{SU}(8)$ indices, it takes the matrix form

$$
W_{i j}=\left(\begin{array}{cc}
-\frac{2}{3} \delta_{\left[k^{[p} W^{q]}\right]_{l] i j}} & \frac{1}{24} \epsilon_{k l r s t u v w} W^{t u v w}{ }_{i j} \\
W^{m n n p q} & \frac{2}{3} \delta_{\left[r^{[m}\right.} W^{n]}{ }_{s] i j}
\end{array}\right)
$$

and complex conjugate. The diagonal and off-diagonal blocks are parametrized by $W^{i}{ }_{j[k l]}$, $W^{[t u v w]}{ }_{i j}$ with $W_{i k l}^{i}=0$. In general, the diagonal and off-diagonal blocks carry the SU(8) representations

$$
\begin{aligned}
W_{j k l}^{i} & : \mathbf{2 8} \oplus \mathbf{3 6} \oplus \mathbf{4 2 0} \oplus \mathbf{1 2 8 0}, \\
W^{t u v w} & : \overline{\mathbf{2 8}} \oplus \overline{\mathbf{4 2 0} \oplus \mathbf{1 5 1 2} .}
\end{aligned}
$$

Together with their complex conjugates they fill the three irreducible $\mathrm{E}_{7(7)}$ representation (A.1). The $\mathbf{3 6} \oplus \overline{\mathbf{3 6}}$ sit in the $\mathbf{9 1 2}$, on the other hand there are two copies of the $420 \oplus \overline{\mathbf{4 2 0}}$ sitting in the $\mathbf{9 1 2}$ and the $\mathbf{6 4 8 0}$, respectively. In order to disentangle the different representations, it is useful to recollect the transformation of $W_{i}{ }^{j k l}, W^{i j k l}{ }_{m n}$ under the $70 \mathrm{E}_{7(7)} / \mathrm{SU}(8)$ generators, which mix these fields as follows [51]

$$
\begin{aligned}
\delta W_{i}{ }^{j k l} & =2 \Sigma^{j m n p} W_{i m n p}{ }^{k l}-\frac{1}{4} \delta_{i}{ }^{j} \Sigma^{m n p q} W_{m n p q}{ }^{k l}+\Sigma^{k l m n} W^{j}{ }_{i m n}, \\
\delta W_{i j k l}{ }^{m n} & =-\frac{4}{3} \Sigma_{p[i j k} W_{l]}{ }^{p m n}-\frac{1}{24} \varepsilon_{i j k l p q r s} \Sigma^{m n t u} W_{t u}^{p q r s} .
\end{aligned}
$$

Iterating this transformation, we can compute the action of the $\mathrm{E}_{7(7)} / \mathrm{SU}(8)$ Casimir $\Delta \equiv \delta_{i j k l} \delta^{i j k l}$

$$
\begin{aligned}
\Delta W_{i}{ }^{j k l} & =\frac{35}{6} W_{i}{ }^{j k l}-\frac{5}{12} W_{m}{ }^{m k l} \delta_{i}{ }^{j}-\frac{1}{2} T_{m n}^{k l m n}{ }_{m i}{ }^{j}-2 \delta_{i}{ }^{[k} W^{l] j m n}{ }_{m n}+2 W^{j k l m}{ }_{i m}, \\
\Delta W^{k l m n}{ }_{i j} & =\frac{11}{6} W^{k l m n}+\frac{4}{3} W_{[i}^{[k l m} \delta_{j]}{ }^{n]}-\frac{4}{3} \delta_{i j}{ }^{[m n} W_{p}{ }^{k l] p}-\frac{1}{3} W_{p}{ }^{p k l} \delta_{i j}{ }^{m n]},
\end{aligned}
$$

whose different eigenvalues allow to identify the $\mathrm{E}_{7(7)}$ origin of the various $\mathrm{SU}(8)$ blocks. E.g. parametrizing the two $\mathbf{4 2 0}$ representations as

$$
W_{i}^{j k l}=A_{i}{ }^{j k l}+\cdots, \quad W^{k l m n}{ }_{i j}=\delta_{[i}^{[k} B_{j]}^{l m n]}+\cdots,
$$

with traceless $A_{i}{ }^{[j k l]}, B_{i}{ }^{[j k l]}$, the action (A.5) diagonalises on the combinations

$$
\begin{aligned}
4 A_{i}{ }^{j k l} & =-3 B_{i}{ }^{j k l}, & & \text { with eigenvalue } \frac{9}{2}, \\
A_{i}{ }^{j k l} & =B_{i}{ }^{j k l}, & & \text { with eigenvalue } \frac{41}{6} .
\end{aligned}
$$


The first option corresponds to the $\mathbf{4 2 0}$ from the $\mathbf{9 1 2}$, cf. (3.28) of [51], the other one thus is the $\mathbf{4 2 0}$ from the $\mathbf{6 4 8 0}$. We conclude, that the projection of (A.6) onto the $\mathbf{4 2 0}$ from the 912 is given by

$$
\frac{3}{7}\left(A_{i}{ }^{j k l}-B_{i}{ }^{j k l}\right)
$$

such that consistently the second combination of (A.7) is projected to zero, and the first one is projected to itself.

Putting everything together, we learn that taking the original spin connection $Q_{A B}{ }^{C}$ living in the $\mathrm{SU}(8)$ of the form (schematically)

$$
Q_{i j}=\left(\begin{array}{c:c}
A_{420}+\ldots & 0 \\
\hdashline 0 & A_{420}+\ldots
\end{array}\right),
$$

i.e. with $B_{i}{ }^{j k l}=0$, its projection onto the $\mathbf{9 1 2}$ maps this into a matrix of the form

$$
\left[Q_{i j}\right]_{(\mathbf{9 1 2})}=\left(\begin{array}{c:c}
\frac{3}{7} A_{420}+\ldots & \frac{3}{7} A_{420}+\ldots \\
\hdashline \frac{3}{7} A_{420}+\ldots & \frac{3}{7} A_{420}+\ldots
\end{array}\right) .
$$

This is the form of the matrix which via (3.18) we identify with the $T$-tensor of gauged supergravity [51], parametrized by $A_{1}, A_{2}$ as in (3.21). Comparing (A.9), (A.10) we see that upon projection, a relative factor of $\frac{3}{7}$ has to be taken into account in the $\mathbf{4 2 0}$ part $A_{2}{ }^{j k l}$, while the $\mathbf{3 6}$ part $A_{1}^{i j}$ (which is unique in (A.3)) remains unchanged.

Open Access. This article is distributed under the terms of the Creative Commons Attribution License (CC-BY 4.0), which permits any use, distribution and reproduction in any medium, provided the original author(s) and source are credited.

\section{References}

[1] T. Kaluza, On the problem of unity in physics, Sitzungsber. Preuss. Akad. Wiss. Berlin (Math. Phys.) 1921 (1921) 966.

[2] O. Klein, Quantum Theory and Five-Dimensional Theory of Relativity. (In German and English), Z. Phys. 37 (1926) 895 [INSPIRE].

[3] E. Cremmer, B. Julia and J. Scherk, Supergravity theory in eleven-dimensions, Phys. Lett. B 76 (1978) 409 [INSPIRE].

[4] E. Cremmer and B. Julia, The SO(8) supergravity, Nucl. Phys. B 159 (1979) 141 [inSPIRE].

[5] B. de Wit and H. Nicolai, $N=8$ supergravity, Nucl. Phys. B 208 (1982) 323 [inSPIRE].

[6] B. de Wit and H. Nicolai, The consistency of the $S^{7}$ truncation in $D=11$ supergravity, Nucl. Phys. B 281 (1987) 211 [INSPIRE].

[7] H. Nicolai and K. Pilch, Consistent truncation of $D=11$ supergravity on $A d S_{4} \times S^{7}$, JHEP 03 (2012) 099 [arXiv:1112.6131] [INSPIRE].

[8] B. de Wit and H. Nicolai, Deformations of gauged $\mathrm{SO}(8)$ supergravity and supergravity in eleven dimensions, JHEP 05 (2013) 077 [arXiv:1302.6219] [INSPIRE]. 
[9] H. Godazgar, M. Godazgar and H. Nicolai, Generalised geometry from the ground up, JHEP 02 (2014) 075 [arXiv: 1307.8295] [INSPIRE].

[10] H. Godazgar, M. Godazgar and H. Nicolai, Nonlinear Kaluza-Klein theory for dual fields, Phys. Rev. D 88 (2013) 125002 [arXiv:1309.0266] [INSPIRE].

[11] B. de Wit and H. Nicolai, $d=11$ supergravity with local $\mathrm{SU}(8)$ invariance, Nucl. Phys. B 274 (1986) 363 [INSPIRE].

[12] H. Nastase, D. Vaman and P. van Nieuwenhuizen, Consistency of the $A d S_{7} \times S^{4}$ reduction and the origin of selfduality in odd dimensions, Nucl. Phys. B 581 (2000) 179 [hep-th/9911238] [INSPIRE].

[13] M. Cvetič, H. Lü and C.N. Pope, Consistent Kaluza-Klein sphere reductions, Phys. Rev. D 62 (2000) 064028 [hep-th/0003286] [INSPIRE].

[14] M. Cvetič, H. Lü, C.N. Pope, A. Sadrzadeh and T.A. Tran, Consistent $\mathrm{SO}(6)$ reduction of type IIB supergravity on $S^{5}$, Nucl. Phys. B 586 (2000) 275 [hep-th/0003103] [InSPIRE].

[15] J. Scherk and J.H. Schwarz, How to get masses from extra dimensions, Nucl. Phys. B 153 (1979) 61 [INSPIRE].

[16] N. Kaloper and R.C. Myers, The odd story of massive supergravity, JHEP 05 (1999) 010 [hep-th/9901045] [INSPIRE].

[17] C.M. Hull, A geometry for non-geometric string backgrounds, JHEP 10 (2005) 065 [hep-th/0406102] [INSPIRE].

[18] A. Dabholkar and C. Hull, Generalised T-duality and non-geometric backgrounds, JHEP 05 (2006) 009 [hep-th/0512005] [inSPIRE].

[19] C.M. Hull and R.A. Reid-Edwards, Gauge symmetry, T-duality and doubled geometry, JHEP 08 (2008) 043 [arXiv: 0711.4818] [INSPIRE].

[20] G. Dall'Agata, N. Prezas, H. Samtleben and M. Trigiante, Gauged supergravities from twisted doubled tori and non-geometric string backgrounds, Nucl. Phys. B 799 (2008) 80 [arXiv:0712.1026] [INSPIRE].

[21] C.M. Hull and R.A. Reid-Edwards, Non-geometric backgrounds, doubled geometry and generalised T-duality, JHEP 09 (2009) 014 [arXiv:0902.4032] [INSPIRE].

[22] W. Siegel, Superspace duality in low-energy superstrings, Phys. Rev. D 48 (1993) 2826 [hep-th/9305073] [INSPIRE].

[23] C. Hull and B. Zwiebach, Double field theory, JHEP 09 (2009) 099 [arXiv:0904.4664] [INSPIRE].

[24] C. Hull and B. Zwiebach, The gauge algebra of double field theory and Courant brackets, JHEP 09 (2009) 090 [arXiv: 0908.1792] [INSPIRE].

[25] O. Hohm, C. Hull and B. Zwiebach, Background independent action for double field theory, JHEP 07 (2010) 016 [arXiv: 1003.5027] [InSPIRE].

[26] O. Hohm, C. Hull and B. Zwiebach, Generalized metric formulation of double field theory, JHEP 08 (2010) 008 [arXiv: 1006.4823] [INSPIRE].

[27] O. Hohm and S.K. Kwak, Frame-like geometry of double field theory, J. Phys. A 44 (2011) 085404 [arXiv: 1011.4101$]$ [INSPIRE].

[28] O. Hohm and S.K. Kwak, Massive Type II in Double Field Theory, JHEP 11 (2011) 086 [arXiv:1108.4937] [INSPIRE].

[29] G. Aldazabal, W. Baron, D. Marques and C. Núñez, The effective action of double field theory, JHEP 11 (2011) 052 [Erratum ibid. 1111 (2011) 109] [arXiv:1109. 0290] [INSPIRE]. 
[30] D. Geissbuhler, Double field theory and $N=4$ gauged supergravity, JHEP 11 (2011) 116 [arXiv: 1109.4280] [INSPIRE].

[31] F. Hassler and D. Lüst, Consistent compactification of double field theory on non-geometric flux backgrounds, JHEP 05 (2014) 085 [arXiv: 1401.5068] [INSPIRE].

[32] M. Graña and D. Marques, Gauged double field theory, JHEP 04 (2012) 020 [arXiv: 1201.2924] [INSPIRE].

[33] O. Hohm and S.K. Kwak, Double field theory formulation of heterotic strings, JHEP 06 (2011) 096 [arXiv:1103.2136] [INSPIRE].

[34] D.S. Berman, E.T. Musaev, D.C. Thompson and D.C. Thompson, Duality invariant M-theory: gauged supergravities and Scherk-Schwarz reductions, JHEP 10 (2012) 174 [arXiv: 1208.0020] [INSPIRE].

[35] E.T. Musaev, Gauged supergravities in 5 and 6 dimensions from generalised Scherk-Schwarz reductions, JHEP 05 (2013) 161 [arXiv:1301.0467] [INSPIRE].

[36] G. Aldazabal, M. Graña, D. Marqués and J.A. Rosabal, Extended geometry and gauged maximal supergravity, JHEP 06 (2013) 046 [arXiv: 1302.5419] [INSPIRE].

[37] D.S. Berman and K. Lee, Supersymmetry for gauged double field theory and generalised Scherk-Schwarz reductions, Nucl. Phys. B 881 (2014) 369 [arXiv:1305.2747] [INSPIRE].

[38] K. Lee, C. Strickland-Constable and D. Waldram, Spheres, generalised parallelisability and consistent truncations, arXiv:1401.3360 [INSPIRE].

[39] W.H. Baron, Gaugings from $E_{7}$ extended geometries, Phys. Rev. D 91 (2015) 024008 [arXiv: 1404.7750] [INSPIRE].

[40] D.S. Berman and M.J. Perry, Generalized geometry and M-theory, JHEP 06 (2011) 074 [arXiv: 1008.1763] [INSPIRE].

[41] D.S. Berman, H. Godazgar, M.J. Perry and P. West, Duality invariant actions and generalised geometry, JHEP 02 (2012) 108 [arXiv: 1111.0459] [INSPIRE].

[42] A. Coimbra, C. Strickland-Constable and D. Waldram, $E_{d(d)} \times \mathbb{R}^{+}$generalised geometry, connections and M-theory, JHEP 02 (2014) 054 [arXiv:1112.3989] [INSPIRE].

[43] A. Coimbra, C. Strickland-Constable and D. Waldram, Supergravity as generalised geometry II: $E_{d(d)} \times \mathbb{R}^{+}$and M-theory, JHEP 03 (2014) 019 [arXiv: 1212.1586] [INSPIRE].

[44] O. Hohm and H. Samtleben, Exceptional form of $D=11$ supergravity, Phys. Rev. Lett. 111 (2013) 231601 [arXiv:1308.1673] [InSPIRE].

[45] O. Hohm and H. Samtleben, Exceptional field theory I: $E_{6(6)}$ covariant form of M-theory and type IIB, Phys. Rev. D 89 (2014) 066016 [arXiv:1312.0614] [INSPIRE].

[46] O. Hohm and H. Samtleben, Exceptional field theory II: $E_{7(7)}$, Phys. Rev. D 89 (2014) 066017 [arXiv: 1312.4542] [INSPIRE].

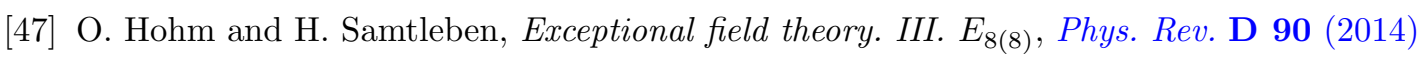
066002 [arXiv: 1406.3348] [INSPIRE].

[48] H. Godazgar, M. Godazgar, O. Hohm, H. Nicolai and H. Samtleben, Supersymmetric E $E_{7(7)}$ exceptional field theory, JHEP 09 (2014) 044 [arXiv: 1406.3235] [INSPIRE].

[49] O. Hohm, S.K. Kwak and B. Zwiebach, Unification of type II strings and T-duality, Phys. Rev. Lett. 107 (2011) 171603 [arXiv:1106.5452] [INSPIRE].

[50] B. de Wit, H. Samtleben and M. Trigiante, On lagrangians and gaugings of maximal supergravities, Nucl. Phys. B 655 (2003) 93 [hep-th/0212239] [INSPIRE]. 
[51] B. de Wit, H. Samtleben and M. Trigiante, The maximal D = 4 supergravities, JHEP 06 (2007) 049 [arXiv: 0705.2101] [INSPIRE].

[52] A. Le Diffon, H. Samtleben and M. Trigiante, $N=8$ supergravity with local scaling symmetry, JHEP 04 (2011) 079 [arXiv:1103.2785] [INSPIRE].

[53] C.M. Hull and N.P. Warner, Noncompact gaugings from higher dimensions, Class. Quant. Grav. 5 (1988) 1517 [inSPIRE].

[54] O. Hohm, D. Lüst and B. Zwiebach, The spacetime of double field theory: review, remarks and outlook, Fortsch. Phys. 61 (2013) 926 [arXiv:1309.2977] [INSPIRE].

[55] D.S. Berman, M. Cederwall, A. Kleinschmidt and D.C. Thompson, The gauge structure of generalised diffeomorphisms, JHEP 01 (2013) 064 [arXiv:1208.5884] [INSPIRE].

[56] E. Bergshoeff, R. Kallosh, T. Ortín, D. Roest and A. Van Proeyen, New formulations of $D=10$ supersymmetry and D8-O8 domain walls, Class. Quant. Grav. 18 (2001) 3359 [hep-th/0103233] [INSPIRE].

[57] M. Cederwall, J. Edlund and A. Karlsson, Exceptional geometry and tensor fields, JHEP 07 (2013) 028 [arXiv: 1302.6736] [INSPIRE].

[58] E. Cremmer, B. Julia, H. Lü and C.N. Pope, Dualization of dualities. 1, Nucl. Phys. B 523 (1998) 73 [hep-th/9710119] [INSPIRE].

[59] A. Le Diffon and H. Samtleben, Supergravities without an action: gauging the trombone, Nucl. Phys. B 811 (2009) 1 [arXiv:0809.5180] [INSPIRE].

[60] J.-H. Park and Y. Suh, U-geometry: SL(5), JHEP 04 (2013) 147 [Erratum ibid. 1311 (2013) 210] [arXiv: 1302.1652] [INSPIRE].

[61] H. Samtleben and M. Weidner, The maximal D $=7$ supergravities, Nucl. Phys. B 725 (2005) 383 [hep-th/0506237] [INSPIRE].

[62] T. Fischbacher, The encyclopedic reference of critical points for $\mathrm{SO}(8)$-gauged $N=8$ supergravity. Part 1: cosmological constants in the range $-\Lambda / g^{2} \in[6: 14.7)$, arXiv:1109.1424 [INSPIRE].

[63] C.M. Hull, The minimal couplings and scalar potentials of the gauged $N=8$ supergravities, Class. Quant. Grav. 2 (1985) 343 [InSPIRE].

[64] C.-h. Ahn and K.-s. Woo, Domain wall and membrane flow from other gauged $D=4, N=8$ supergravity. Part 1, Nucl. Phys. B 634 (2002) 141 [hep-th/0109010] [INSPIRE].

[65] G. Dall'Agata and G. Inverso, De Sitter vacua in $N=8$ supergravity and slow-roll conditions, Phys. Lett. B 718 (2013) 1132 [arXiv:1211.3414] [InSPIRE].

[66] M. Cvetič, H. Lü, C.N. Pope, A. Sadrzadeh and T.A. Tran, $S^{3}$ and $S^{4}$ reductions of type IIA supergravity, Nucl. Phys. B 590 (2000) 233 [hep-th/0005137] [INSPIRE].

[67] S. Deger, A. Kaya, E. Sezgin and P. Sundell, Spectrum of $D=6, N=4 b$ supergravity on AdS $S_{3}$ times $S^{3}$, Nucl. Phys. B 536 (1998) 110 [hep-th/9804166] [INSPIRE].

[68] N.S. Deger, H. Samtleben, O. Sarioglu and D.V.d. Bleeken, A supersymmetric reduction on the three-sphere, Nucl. Phys. B 890 (2014) 350 [arXiv:1410.7168] [INSPIRE].

[69] O. Hohm, W. Siegel and B. Zwiebach, Doubled $\alpha^{\prime}$-geometry, JHEP 02 (2014) 065 [arXiv: 1306.2970] [INSPIRE].

[70] O.A. Bedoya, D. Marques and C. Núñez, Heterotic $\alpha^{\prime}$-corrections in double field theory, JHEP 12 (2014) 074 [arXiv:1407.0365] [INSPIRE].

[71] O. Hohm and B. Zwiebach, Green-Schwarz mechanism and $\alpha^{\prime}$-deformed Courant brackets, arXiv: 1407.0708 [INSPIRE]. 
[72] O. Hohm and B. Zwiebach, Double field theory at order $\alpha^{\prime}$, JHEP 11 (2014) 075 [arXiv: 1407.3803] [INSPIRE].

[73] A. Coimbra, R. Minasian, H. Triendl and D. Waldram, Generalised geometry for string corrections, JHEP 11 (2014) 160 [arXiv:1407.7542] [INSPIRE].

[74] L.B. Anderson, J.Gray and E.Sharpe, Algebroids, heterotic moduli spaces and the Strominger system, JHEP 07 (2014) 037 [arXiv:1402.1532] [INSPIRE].

[75] X. de la Ossa and E.E. Svanes, Connections, field redefinitions and heterotic supergravity, JHEP 12 (2014) 008 [arXiv: 1409.3347] [INSPIRE]. 